\title{
Context-dependent decision making in a premotor circuit
}

\section{Authors}

4 Zheng $\mathrm{Wu}^{1,2}$, Ashok Litwin-Kumar ${ }^{2}$, Philip Shamash ${ }^{2,3}$, Alexei Taylor ${ }^{1,2}$, Richard Axel ${ }^{1,2^{*}}$,

$5 \quad$ Michael N. Shadlen ${ }^{1,2,4 *}$

6

\section{$7 \quad$ Affiliations}

$8 \quad{ }^{1}$ Howard Hughes Medical Institute, Columbia University, New York, NY 10027, USA.

$9{ }^{2}$ Mortimer B. Zuckerman Mind Brain Behavior Institute and Department of Neuroscience,

10 Columbia University, New York, NY 10027, USA.

$11{ }^{3}$ Present address: Sainsbury Wellcome Centre, University College London (UCL), London, UK.

$12{ }^{4}$ Lead Contact

13

14 *Correspondence to R.A. (ra27@ columbia.edu) or M.N.S. (shadlen@ columbia.edu) 


\section{Summary}

18 Cognitive capacities afford contingent associations between sensory information and behavioral

19 responses. We studied this problem using an olfactory delayed match to sample task whereby a

20 sample odor specifies the association between a subsequent test odor and rewarding action. Multi-

21 neuron recordings revealed representations of the sample and test odors in olfactory sensory and

22 association cortex, which were sufficient to identify the test odor as match/non-match. Yet,

23 inactivation of a downstream premotor area (ALM), but not orbitofrontal cortex, confined to the

24 epoch preceding the test odor, led to gross impairment. Olfactory decisions that were not context

25 dependent were unimpaired. Therefore, ALM may not receive the outcome of a match/non-match

26 decision from upstream areas but contextual information - the identity of the sample — to establish

27 the mapping between test odor and action. A novel population of pyramidal neurons in ALM layer

282 may mediate this process.

\section{Keywords}

31 Decision-making, flexible behavior, context, working memory, premotor cortex, olfaction 


\section{Introduction}

35 Brain functions deemed "cognitive" exhibit complexities that extend an organism's behavioral

36 repertoire beyond simple sensory-response associations, motor programs, and instructed actions.

37 Cognitive functions exploit contingent, hierarchical processes of decision making and executive

38 control. For example, instead of choosing an action, a decision might lead an organism to choose

39 a strategy, or to switch tasks, or to make yet another decision. Cognitive functions may also

40 transpire over flexible time scales without precipitating an immediate behavior, as when a decision

41 is based on information acquired at some moment and combined with information acquired later.

43 Neural mechanisms of decision making and executive control have been studied primarily in the

44 parietal and prefrontal cortex of nonhuman primates. These studies focused mainly on neurons that

45 exhibit responses over long time-scales and implicated processes such as working memory

46 (Funahashi et al., 1989; Fuster and Alexander, 1971), planned action (Cisek and Kalaska, 2005;

47 Evarts and Tanji, 1976; Snyder et al., 1997), behavioral state (Harvey et al., 2012; Kadohisa et al.,

48 2013), representation of stimulus qualities (Freedman and Assad, 2006; Romo et al., 1999), and

49 reasoning (Gold and Shadlen, 2007; Yang and Shadlen, 2007). Thus the study of the neural

50 mechanisms of perceptual decision making may provide an initial logic for higher order cognition.

51 This will require an understanding of neural circuits at a level that is not yet possible to achieve in

52 nonhuman primates. Thus there has been growing interest in the pursuit of elementary cognitive

53 functions in the mouse, for which genetic and viral tools for circuit manipulation abound

54 (Carandini and Churchland, 2013; Luo et al., 2018). The challenge is to find simple behaviors,

55 within the mouse repertoire, that have the potential to elucidate more complex cognitive functions. 
57 We developed a simple task that allows us to study rudiments of executive control and decision-

58 making in a mouse. The task is a variant of the logical XOR problem, realized as an olfactory

59 delayed match to sample task (DMS; Fig. 1) (Liu et al., 2014). The mouse is exposed to a sample

60 odor, either $\mathrm{S}_{\mathrm{A}}$ or $\mathrm{S}_{\mathrm{B}}$, and, after a short delay, receives either $\mathrm{A}$ or $\mathrm{B}$ as a test order. To receive a

61 reward, the mouse must lick to the left if the sample and test odors are the same and to the right if

62 they are different. We reasoned that mice could perform this task by interpreting the sample odor

63 as an instruction. The instruction is to select the appropriate association between the test odor and

64 the correct behavioral response - a left or right lick.

66 We hypothesized that this hierarchical control might be solved by changing the configuration of

67 cortical circuitry in the premotor cortex, area ALM. Studies from Svoboda and colleagues have

68 shown that ALM plays an essential role in behaviors in which a sensory cue serves as an instruction

69 to lick to the left or right (Guo et al., 2014; Li et al., 2015; Svoboda and Li, 2018). They showed

70 that many neurons in ALM can hold such an instruction — or the plan to lick to the left or right-

71 in persistent activity through a delay period. In the DMS task we study, the sample odor cannot

72 provide an instruction to lick left or right, but only a context to interpret the test odor. Thus the

73 mouse cannot plan a lick until the arrival of the test odor after the delay. We investigated three

74 hypotheses for the involvement of ALM in this task. First, areas upstream to ALM could solve the

75 match/non-match discrimination and project this solution to ALM which organizes an appropriate

76 lick response. Second, ALM could decode upstream representations that combine sample and test

77 odors to select the appropriate response. Third, ALM could receive information about identity of

78 the sample odor to instantiate the appropriate response to the test odor. We provide experimental 
79 evidence for this third possibility. We show that the sample odor is represented in ALM itself, and

80 that this representation allows ALM to associate the test odor with the appropriate lick response.

\section{Results}

83 Mice were trained to compare a sample and test odor, separated by a delay and to report their

84 decisions of "match" or "non-match" by licking to the left or to the right, respectively (Fig. 1 A).

85 The lick-left, lick-right design requires distinct actions to report both match and non-match.

86 Importantly, animals cannot solve the task until they smell the test odor. For most experiments, we

87 used the same two odors, (+)- $\alpha$-Pinene and cis-3-Hexen-1-ol (odors A and B, respectively) on all

88 trials. Animals were trained to suppress their premature licks before test onset (Fig. 1D) and do

89 not appear to use licks to represent and remember the sample odors (Fig. S1E-G).

91 Mice performed the task with a median accuracy of $90 \%(n=41$; Fig. $1 \mathrm{C})$. When challenged with

92 a novel pair of odorants after training with pinene and hexenol, mice performed at chance level

93 with a high no-choice rate (Fig. S1C). This suggests that the mice failed to generalize the

94 match/non-match rule. Rather they learned that test odor $T_{A}$ instructs "lick left" if preceded by

95 sample odor $S_{A}$ (AA trial), whereas it instructs "lick right" if preceded by sample odor $S_{B}$ (BA

96 trial). The complementary logic holds for the test odor $T_{B}$ (Fig. 1B). This flexible association

97 between test odor and licking response is contingent on the sample odor identity, which must be

98 represented through the delay in order to affect the match/non-match decision.

100 We first characterize the neural representation of the sample and test odors by surveying neural 101 responses in the Piriform cortex (Pir), the orbitofrontal cortex (OFC) and the anterolateral motor 
cortex (ALM) while the mouse performed the DMS task. We wished to determine whether each of these areas contain a persistent representation of the sample odor and whether they encode the test odor in a way that might inform the match-nonmatch decision. As detailed in the next section, the findings support the possibility that match/non-match decision can be established within Pir and OFC and then transmitted to the ALM, to render the lick response. We then test this model by

107 inactivating ALM during the sample and delay periods of the task. We find evidence against this model and instead establish a necessary role of ALM during the sample and delay periods. Finally, using 2-photon Ca imaging, we expose a class of neurons in superficial ALM that could mediate the circuit changes required to allow ALM to associate the test odor with an appropriate lick

111 response.

\section{Representations of sensory and motor signals in Pir, OFC and ALM}

114 We performed electrophysiological recordings in brain areas likely to be involved in the DMS task, piriform cortex (Pir), orbitofrontal cortex (OFC), and ALM. Pir is a primary olfactory sensory area

116 (Giessel and Datta, 2014; Sosulski et al., 2011) that encodes odor identity. Pir projects to OFC, a

117 higher-order associative area that is thought to encode value, expectation, and working memory

118 (Bechara et al., 2000; Mainen and Kepecs, 2009; Padoa-Schioppa and Assad, 2006; Ramus and

119 Eichenbaum, 2000). Figure 2 provides examples of neurons that respond selectively to $(i)$ one or

120 the other sample odor during the sample- and delay-periods ( $1^{\text {st }}$ and $2^{\text {nd }}$ columns); (ii) one or the

121 other test odor ( $3^{\text {rd }}$ column); and (iii) one or the other choice ( $4^{\text {th }}$ column). It also shows the strength

122 of the selectivity across all the neurons recorded. The selectivity index quantifies the degree to

123 which the distributions of firing rates to the two conditions (e.g., odor A or B) are non-overlapping

124 (see Methods). In what follows we refer to a neuron as selective if the difference in the mean 
125 responses is statistically reliable (Mann-Whitney $\mathrm{U}$ test, $\mathrm{p}<0.01$, two-tailed, uncorrected; indicated 126 by shading in Fig. 2D-G).

128 More than a third of neurons (37\%) in Pir responded selectively to either odor A or odor B during 129 the sample epoch (Fig. 2A, D). Of these, 71\% exhibited the same odor preference during the 130 sample and test epochs (Fig. S2A) suggesting that these neurons represent sensory information 131 and encode odor identity. Most of the sample-selective neurons responded transiently (e.g., Fig.

132 2A, left most panel), but $28 \%$ exhibited persistent selectivity through the delay (e.g., Fig. 2A, $2^{\text {nd }}$

133 panel from left; Fig. S2D). These neurons can therefore inform downstream neurons of the identity

134 of the sample odor at the time of test. A subset of Pir neurons responded to the test odor in a way 135 that depended on the identity of the sample odor (Fig. S2G; Methods). These neurons are 136 characterized as trial type selective (6.2\% of all Pir neurons). Finally, $4.5 \%$ of Pir neurons 137 exhibited selective responses during the test epoch that reflect either a match or non-match between 138 sample and test odors (Fig. 2A, G). The information represented by these last two classes of 139 neurons, trial type and match/non-match, would appear to be sufficient to guide motor output in 140 downstream brain areas.

142 In OFC, $24.5 \%$ of the neurons were odor selective during the sample epoch (Fig. 2B, D) and 60\% 143 of these exhibited the same odor preference at test (Fig. S2B), suggesting that they encode odor 144 identity. Compared to Pir, fewer of the sample-selective neurons in OFC exhibited persistent 145 spiking through the delay epoch (Fig. S2E; $13 \%$ in OFC versus $28 \%$ in Pir). Only $3.7 \%$ of the 146 neurons were selective to trial type (Fig. S2H), but a greater fraction (11\%) responded selectively 
147 to match/non-match trials (Fig. 2G). Therefore, the OFC also appears to have integrated sensory

148 inputs in a way that could guide appropriate motor output.

150 Unlike Pir and OFC, which largely represent odor identity, the dominant task related activity in

151 ALM was correlated with the lick response - the outcome of the match/non-match decision; $28 \%$

152 of ALM neurons distinguished match from non-match trials during the test epoch (Fig. 2C, G).

153 Analyses of error trials and premature licks show that the neural activity reflects lick direction

154 rather than the identities of the sample and test odors or the true match/non-match condition,

155 consistent with the role of ALM in motor planning (Fig. S2J, K) (Guo et al., 2014; Li et al., 2015;

156 Svoboda and Li, 2018). A largely non-overlapping population in ALM (12\%) exhibited weak odor

157 selectivity during the sample epoch, and among these, $12 \%$ (less than $1.5 \%$ overall) maintained

158 this selectivity through the end of the delay epoch (Fig. 2D, S2F). Very few neurons exhibited a

159 selective response to the test odor, and only $1.4 \%$ of ALM neurons were trial type selective (Fig.

160 S2I). Together, these recordings are consistent with the known role for ALM in the preparation of

161 an appropriate motor response (Svoboda and Li, 2018) following the match/non-match decision.

163 This survey of a sensory, association and premotor cortex reveals neurons in each area that exhibit

164 one or more of the properties required to solve the DMS task: ( $i$ ) a representation of the sample

165 odor during the delay epoch and (ii) a response to the test odor that is possibly modulated by the

166 identity of the sample odor and (iii) the conversion of these sensory signals to choice-related

167 activity. Figure 3A shows the averaged difference in firing rate to each neuron's preferred versus

168 nonpreferred odor during the trial. The assignment of preferred odor was derived from five

169 randomly selected trials to each odor, which are excluded from the averages. The procedure 
170 ensured an unbiased estimate of the difference (see Methods). The average difference is strongest

171 in Pir and weakest in ALM. In the left panel, the magnitude of the difference reflects a combination

172 of greater selectivity and the fraction of neurons that are selective, as nonselective neurons drive

173 the average toward zero. In the right panel, the averages comprise only the selective cases from

174 each area (filled histograms in Fig. 2). All three areas contain signals that could convey the

175 representation of the sample odor ( $\mathrm{Pir}>\mathrm{OFC}>\mathrm{ALM})$. However, this conclusion is based on 176 averaged activity across trials.

177

178 To determine the information available about task variables present in population responses, we

179 trained linear classifiers to decode these variables from simultaneously recorded neurons on single

180 trials (from 14 to 120 simultaneously recorded neurons; see Methods). As shown in Figure 3B, all

181 three areas contain signals that support classification of the sample odor above chance levels

182 throughout the sample and delay periods. The performance of the classifier is lower for ALM

183 recordings compared to Pir recordings, for which the sample odor identity can be decoded

184 throughout the delay period and into the test epoch. This extended sample-selective response could

185 potentially be used to perform a comparison between sample and test odors. Consistent with this,

186 classification of all four trial types is possible using the Pir recordings (Fig. 3C). In this same epoch

187 (test odor onset to the first lick response), it is possible to decode the binary choice from all three

188 areas, with ALM exhibiting the highest performance (Fig. 3D).

190 The neural recordings and decoding analyses would appear to support a traditional hierarchical

191 view of the mechanism responsible for resolving the match/non-match decision. That is, the 192 expression of the decision by the left- and right-lick neurons in ALM could arise by reading out 
193 activity in OFC or Pir, perhaps via another intermediate area between Pir and ALM. On this view, 194 area ALM is only essential once the test odor arrives, when it must either $(i)$ convert the decision 195 to a lick response or (ii) perform a computation, similar to our decoder, to convert the population 196 response from upstream areas to a left or right lick response. Both mechanisms lead to the 197 prediction that inactivation of ALM during the sample and delay periods should not impair 198 performance on the task. As we next show, this seemingly obvious prediction is incorrect.

200 ALM is required for the match/non-match computation.

201 We inactivated ALM bilaterally on a fraction of the trials by photostimulation of GABAergic 202 interneurons expressing channelrhodopsin-2 (ChR2) (Fig. 4A, S3; Methods) (Guo et al., 2014; 203 Zhao et al., 2011). Inactivation was confined to the sample and delay epochs and was tapered 204 gradually at the end of the delay (see Methods) such that by the onset of the test odor, ALM should

205 be capable of receiving information from upstream sensory and association areas. We reasoned 206 that if ALM reads out the DMS decision computed in upstream areas then inactivation of ALM 207 before the arrival of the test odor should not impair performance.

209 Contrary to predictions, bilateral inactivation of ALM markedly impaired performance on the 210 DMS task (Fig. 4B). The proportion of correct trials decreased from 0.86 on the control trials to 2110.68 (0.5 reflects chance performance). In contrast, bilateral inactivation of OFC induced no 212 impairment on the same task (proportion correct: 0.82 and 0.84 with and without inactivation; Fig. 213 4C). Photostimulation of ALM in animals that did not express ChR2 did not diminish performance 214 (Fig. 4D). Inactivation of ALM caused less impairment when it was restricted to only a portion of 215 the sample plus delay epochs (Fig. 4E). Inactivation during the late delay reduced the proportion 
216 of correct trials from 0.86 to 0.79 , whereas inactivation during the sample and early delay reduced

217 the proportion to 0.83 . These more modest effects suggest that the impairment can be ameliorated

218 when there is a time window in which ALM can receive task related information.

220 The behavioral impairment induced by ALM inactivation is a consequence of altered activity

221 within ALM itself during the sample and delay. Photoinactivation of ALM did not affect odor or

222 match/non-match selectivity in upstream areas, Pir and OFC. During inactivation, the population

223 odor selectivity in Pir or OFC did not change appreciably (Fig. 4J, K, M, N) and our ability to

224 decode match vs non-match from activity was similarly unchanged (Fig. 4Q, R). Neural recordings

225 from ALM during silencing demonstrated that optogenetic suppression eliminated the

226 representation of the sample odor, but neural activity returns after the laser was ramped down (Fig.

227 4I, L, O, P). Despite their diminished performance, animals invariably licked to one of the two

228 ports in inactivation trials, and there was no consistent change in the reaction time (Fig. S4A, B).

229 From these observations, we conclude that the impairment was not explained by a loss of

230 processing capacity in Pir or OFC or from physiological sequelae of photoinactivation of ALM

231 that might affect its function during the test epoch. This last conclusion deserves further scrutiny.

233 A possible concern is that the inactivation of ALM during sample and delay epochs disrupts the

234 ALM circuitry such that it is unable to process information about the test odor or to receive

235 information about the decision from an upstream area. We evaluated this possibility with two

236 control experiments that required the mice to lick to the left or right, based on the identity of a test

237 odor, but did not require a comparison of the test odor to the sample odor. In the first control, an

238 uninformative sample odor A was presented on all trials, but the mouse was rewarded for licking 
left or right for test odor $\mathrm{A}$ or $\mathrm{B}$, respectively ( $\mathrm{AA}$ and $\mathrm{AB}$ trials; Fig. 4A). Inactivation during the sample and delay epochs produced a small impairment (0.91 to 0.88 correct; Fig. $4 \mathrm{~F})$ that was much less pronounced than on the DMS trials (Fig. 4H, green vs. dark blue, $\mathrm{p}<0.0001$; Methods).

243 In the second control, we incorporated two additional trial types into the $\mathrm{AB} \times \mathrm{AB}$ design using

244 two new odors (C and D). In these CC and CD trials, as in the previous control, the correct lick 245 behavior was determined only by the test odor. The six trial types were randomly interleaved (Fig. 246 4A). Inactivation of ALM during the sample and delay epochs led to minimal impairment in CC 247 and CD trials (0.94 to 0.91 correct), whereas significant impairment was replicated in the 248 interleaved DMS trials (0.82 to 0.68 correct; Fig. 4G). Further, the laser introduced no side bias in 249 the $\mathrm{CC} / \mathrm{CD}$ control $\left(\beta_{2}=-0.46\right.$, s.e.m. $=0.40, P=0.25$, Equation 8; see Methods $)$, demonstrating 250 that the bias observed in some of the DMS trials is not due to unbalanced inactivation of the two 251 hemispheres (see also Fig. S4C). These control experiments demonstrate that after recovery from 252 inactivation, ALM is capable of processing information that instructs a licking response via a 253 simple association between two odors and two actions. The impairment on the DMS task must 254 therefore arise by interfering with the process that allows the sample odor to establish the 255 association on each trial. Moreover, it implies that this process occurs in ALM.

\section{Enrichment of sample-selective neurons in ALM Layer 2}

258 Based on the neural recordings, the necessity of ALM during the sample and delay seems highly

259 perplexing. Less than $1.5 \%$ of neurons in ALM had activity that was informative about the identity

260 of the sample. We considered that we might have missed neurons, especially from superficial 261 cortical layers (Fig. S2L; see also Guo et al., 2017, their Fig. 3D). We therefore examined neural 
262 responses in ALM with 2-photon calcium imaging while mice performed the DMS task. Imaging

263 was performed in mice expressing the calcium indicator GCaMP6f in pyramidal cells (Chen et al.,

264 2013; Madisen et al., 2015) (Methods). Consistent with our electrical recordings, calcium imaging

265 revealed that $\sim 37 \%$ of the neurons were choice selective across all cortical depths examined (Fig.

$2665 \mathrm{G}, \mathrm{S} 5 \mathrm{~A}, \mathrm{D})$.

267

268 However, we also observed a large subset of neurons with striking odor selectivity during the

269 sample and delay epoch (Fig. 5A, S5B, C). Interestingly, the odor selective calcium activity was

270 heterogeneous in its timing during the sample and delay epochs, showing a variety of latencies and

271 time scales (Fig. 5B-E). Some of the signals spanned the sample and part of the delay period,

272 peaking at consistent times across trials (long duration, low scatter), spanning the sample and delay

273 period (e.g., Fig. 5C). Other neurons exhibited more transient responses that occurred at different

274 times across trials (short duration, high scatter; e.g., Fig. 5D). Although they are ordered on the

275 graph for visualization, these brief, scattered activations appeared random. For example, their

276 timing is uncorrelated in simultaneously recorded neurons (Fig. S6A, B). These characterizations

277 also hold for the estimated spike rates, achieved through deconvolution of the raw Ca signals (Fig.

278 S6C-F), despite a revision of the estimates of signal duration. From the imaging data, we adduce

279 that a subpopulation of ALM neurons represents the identity of the sample odor through the sample

280 and delay period.

281

282 These sample selective neurons appear to constitute a distinct cell type. They were concentrated

283 in the superficial layer 2 (L2; 100-200 $\mu \mathrm{m}$ from the pial surface), where $18 \%$ responded selectively

284 to one or the other the sample odor (Fig. 5A, F). They were only rarely encountered between 200 
$\mu \mathrm{m}$ and $600 \mu \mathrm{m}$ below the pial surface $(P<0.05$, Kruskal-Wallis test with Tukey-Kramer multiple comparison). This layer specificity is not explained by an inability to measure calcium signals in deeper layers, as comparable fractions of choice-selective cells were found at all depths examined $(P=0.49$, Kruskal-Wallis test; Fig. 5H, S5D). Indeed, a decoder, trained to classify the sample odor, performs much better using L2 neurons than using neurons from the other layers we sampled (Fig. 6A). In contrast, the capacity to decode choice is similar across all layers (Fig. 6B). Most of these sample selective neurons did not respond to the test odor (87 of 108, 81\%; Fig. 6C) and only a few were choice selective or active during licking (Fig. 6D). Their time course distinguishes them from the rare odor selective responses encountered in our electrical recordings, and the absence of lick and choice activity distinguishes them from the dominant cell type in ALM.

297 of the sample odor throughout the delay period. It is therefore possible that the match/non-match decision is made within ALM, based on two external inputs that convey $(i)$ the identity of the sample odor during the sample or delay period and (ii) and the identity of the test odor after the delay. Indeed, the activity of the L2 neurons during the sample and delay is informative about whether the mouse will ultimately succeed in the trial by making the correct choice. The logistic analysis (Fig. 6E) shows that when neurons selective for sample odor A, say, responded less to sample odor A or more strongly to sample odor B, the mouse was more likely to make an erroneous response. Such single trial correlations between neural response and choice, termed choice probability, have been exploited in perceptual decisions to support the proposal that a neuron's response contributes to the decision process, either directly or via correlation with other neurons 
308 or lick left/right decision. Rather, it is a decision about which mapping to apply from test odors to

309 lick responses (Fig. 1B).

\section{Potential mechanisms}

312 We do not yet know the mechanism by which a representation of the sample odor in ALM affects

313 the match/non-match decision, but it appears that some process requiring ALM in the sample and

314 delay period must establish a state at the time of test that can implement the correct mapping

315 between externally derived signals about the identity of the test odor and the activation of the

316 appropriate lick neurons. Several possible mechanisms could underlie the formation of such a state.

317 One possibility is that the state is defined by the persistent firing of sample-selective neurons as in

318 standard "attractor" models of working memory (Fig. 7A) (Amit and Brunel, 1997; Goldman-

319 Rakic, 1995). In this model, the requirement of ALM during the sample and delay epochs (Fig. 4)

320 demands that ALM itself maintain a persistent representation of the sample odor. If the persistent

321 sample representation is maintained in upstream areas but not ALM, the network model predicts

322 that inactivating ALM would cause little behavioral impairment (Fig. 7B, top trace). This is

323 inconsistent with our experimental observation. If the persistent sample representation is only

324 maintained in ALM, the network model predicts that inactivation during the sample and early

325 delay epochs should produce gross impairment (Fig. 7B, bottom trace). This too is inconsistent

326 with the data (Fig. 4E). The weaker effects of inactivation when it was restricted to portions of the

327 sample and delay epochs suggest that ALM's representation of the sample odor can be partially

328 recovered by activity in upstream areas. (Liu et al., 2014)(Liu et al., 2014)Thus, our experimental

329 results argue for models in which multiple brain regions including both ALM and additional

330 upstream areas each maintain a persistent representation of the sample odor (Fig. 7B, middle trace). 
332 The attractor models posit that a representation of the sample odor is maintained through the

333 persistent firing of neuronal assemblies. However, this memory might be maintained in other ways.

334 We observed that impairment was more profound when ALM was silenced during the entire

335 sample and delay epochs compared with inhibition only late in the delay (Fig. 4E). This suggests

336 that a trace of the sample odor identity can persist in ALM during silencing. This trace may be

337 maintained by facilitation or depression of synapses formed by axons of odor-selective neurons

338 onto lick-left or lick-right neurons (Mongillo et al., 2008) (Fig. 7C). It could also be achieved

339 through dendritic gating mechanisms that selectively route test odor information depending on the

340 sample odor identity (Yang et al., 2016) (Fig. 7D). In this model, neurons selective for a particular

341 sample odor enhance or suppress dendritic branches of lick-selective neurons where either A or B

342 inputs concentrate. This synaptic modulation could be controlled by sample-selective neurons in

343 ALM layer 2.

345 Discussion

346 Decisions that engage cognitive capacities entail at least three elemental processes: (i) the

347 maintenance of information over time scales that can extend for seconds prior to a response; (ii)

348 the association of the same information with different responses; and (iii) the association of the

349 same response with different information. The first process requires planning and working memory

350 (Fuster, 1973; Goldman-Rakic, 1995; Romo et al., 1999), whereas the latter two involve flexible, 351 context dependent routing of information to appropriate outputs. 
353 Our findings suggest an unexpected role of ALM in processing perceptual information. Neurons

354 in ALM are known to play an essential role in the planning of licking (Svoboda and Li, 2018). In

355 primates and more recently in rodents, preparatory activity in the premotor parietal circuit has been

356 shown to play a role in perceptual decisions in which attributes of sensory stimuli (e.g., the

357 direction of moving random dots) instruct the selection of a movement (Hanks et al., 2015; Shadlen

358 and Newsome, 2001). In this behavioral paradigm, movement selection evolves gradually and

359 reflects the deliberation process leading to a decision (Gold and Shadlen, 2003; Selen et al., 2012;

360 Spivey et al., 2005). These observations might suggest that premotor area would play a role in the

361 perceptual decision in our DMS task. However, in our task a plan to lick left or right cannot be

362 prepared until the test odor arrives. What can be prepared is the sensory-motor association between

363 the test odor and the appropriate lick direction. Therefore, the impairment in performance arising

364 from the inactivation of ALM does not reflect a disruption of movement preparation but of the

365 state of the circuit that readies it to process information about the test odor and select the 366 appropriate lick response.

368 Previous studies have demonstrated persistent activity in ALM in association with perceptual 369 decision making. For example, elevated activity was reported in direction selective lick neurons 370 when mice associated different haptic stimuli (applied to a whisker) with a lick to the left or right

371 (Chen et al., 2017; Guo et al., 2014; Li et al., 2015; Svoboda and Li, 2018). This persistent activity

372 is involved in planning and driving movement based on a sensory-response association. In our

373 study, on the other hand, persistent activity in sample-selective neurons represents a sensory

374 category rather than a plan of action, because the action cannot be selected until after the delay 375 period upon receipt of the test odor. Hence, unlike in a sensory-instructed delayed response task 
376 (Guo et al., 2014; Li et al., 2015) in which persistent activity represents decision outcome, the

377 activity of our L2 neurons is not the outcome of the DMS decision because this decision has yet

378 to be made. This activity could be viewed as representing an intermediate decision about which

379 mapping from test odor to lick response to employ (Fig. 1B). It is possible that our sample selective

380 neurons overlap those reported previously, but we suspect the overlap is minimal. The persistent

381 activity we observed was concentrated primarily in superficial L2 neurons that did not exhibit lick

382 responses, whereas the persistent activity reported in the somatosensory instructed delay task was

383 found in all cortical layers and in many neurons that responded to error responses in a way that 384 agrees with their role in motor planning.

386 We considered several alternative explanations of the impairment produced by ALM inactivation.

387 The control experiments rule out a simple motor impairment or motor bias, allowing us to focus

388 on the delay period activity. Our interpretation is that the representation of the sample odor in

389 ALM establishes a state of the ALM circuit such that it can perform a simple sensory-response

390 association at the time of test. An alternative is that ALM must prepare both potential motor plans

391 during the delay period (Cisek and Kalaska, 2005) in order for one to be selected by an instruction

392 from another brain area, and it is solely the disruption of this preparatory activity that explains the

393 impairment. This account predicts incorrectly that inactivation would also impair performance on

394 the $\mathrm{A} \times \mathrm{A} / \mathrm{B}$ and $\mathrm{C} \times \mathrm{C} / \mathrm{D}$ tasks. It is also inconsistent with the neural recordings, which failed to

395 reveal simultaneous preparation of the two lick directions during the delay period.

397 Another alternative explanation is that inactivation before the test period disrupts a general state 398 of readiness in ALM to respond to any signal instructing an action to be performed. We find this 
unlikely, because to account for our behavioral observations, this state would need to be specific to the DMS task and not control tasks with identical temporal structures and actions. Finally, we note that DMS is a more difficult task than the controls. Therefore, it may be argued that DMS is more susceptible to perturbations. However, it is not the motor action that makes DMS more difficult than the controls, because all the tasks require the identical motor responses. If the only

404 role of ALM is motor preparation, then inactivating ALM should not result in differential 405 impairment of the DMS and control tasks (Fig. 4H). More importantly, our perturbation affected 406 ALM before a decision could be prepared, because the decision cannot be made before the test 407 odor arrives. Previous studies of decisions to lick to the left or right examined only the preparatory 408 phase after the decision was instructed (Guo et al., 2014; Li et al., 2015).

410 Neurons in superficial layers of the neocortex receive input from controlling structures such as 411 intralaminar and accessory thalamic nuclei (Jones, 1998) as well as long range feedback, e.g., 412 perirhinal cortex to ALM (Zingg et al., 2014). These layers contain the distal dendrites of 413 projection neurons in deeper layers, and activation/suppression of these dendrites can alter the 414 functional properties of the projection neurons (Bittner et al., 2017; Larkum et al., 1999, 2009; 415 Polsky et al., 2004). Thus, it is possible that the selectivity to the sample odor that we observed in 416 L2 neurons might reflect a general mechanism that alters the state of ALM circuitry to flexibly 417 implement different sensorimotor mappings. It remains to be seen if the L2 neurons are in fact 418 critical and if so, how they alter the state of the ALM circuit. However, the association between 419 the probability of an error and the level of activity on single trials (Fig. 6K) suggests they play a 420 functional role. 
422 It may seem counterintuitive that a premotor area would play a critical role in a decision about the

423 relationship between two sensory stimuli. However, the ultimate goal of both sensory and motor

424 systems is not to identify stimulus features or categories, but to determine whether to act one way

425 or another. A potential action might require support from different sources of sensory input. Thus,

426 decisions to act - even if only provisionally — rest on a capacity of the motor system to establish

427 functional connections that allow it to integrate different sources of sensory information, and this

428 information infiltrates the responses of neurons in cortical areas that are appropriately designated

429 premotor or associative (Cisek, 2011; Shadlen and Kiani, 2013). These functional connections

430 must be rapidly modifiable as the demands of an organism and its context change. This capacity

431 for dynamic circuit configuration is likely to support a wide range of cognitive phenomena

432 involving flexible routing, decision making, and perceptual inference (Shadlen and Shohamy, 433 2016). 


\section{References}

437 Amit, D.J., and Brunel, N. (1997). Model of global spontaneous activity and local structured

438 activity during delay periods in the cerebral cortex. Cereb. Cortex 7, 237-252.

439 Bechara, A., Damasio, H., and Damasio, A.R. (2000). Emotion, Decision Making and the

440 Orbitofrontal Cortex. Cereb. Cortex 10, 295-307.

441 Bittner, K.C., Milstein, A.D., Grienberger, C., Romani, S., and Magee, J.C. (2017). Behavioral

442 time scale synaptic plasticity underlies CA1 place fields. Science (80-. ). 357, 1033-1036.

443 Carandini, M., and Churchland, A.K. (2013). Probing perceptual decisions in rodents. Nat.

444 Neurosci. 16, 824-831.

445 Chen, T.W., Wardill, T.J., Sun, Y., Pulver, S.R., Renninger, S.L., Baohan, A., Schreiter, E.R.,

446 Kerr, R.A., Orger, M.B., Jayaraman, V., et al. (2013). Ultrasensitive fluorescent proteins for

447 imaging neuronal activity. Nature 499, 295-300.

448 Chen, T.W., Li, N., Daie, K., and Svoboda, K. (2017). A Map of Anticipatory Activity in Mouse

449 Motor Cortex. Neuron 94, 866-879.e4.

450 Cisek, P. (2011). Cortical mechanisms of action selection: the affordance competition

451 hypothesis. Model. Nat. Action Sel. 208-238.

452 Cisek, P., and Kalaska, J.F. (2005). Neural correlates of reaching decisions in dorsal premotor

453 cortex: Specification of multiple direction choices and final selection of action. Neuron 45, 801-

454814.

455 Evarts, E. V., and Tanji, J. (1976). Reflex and intended responses in motor cortex pyramidal tract

456 neurons of monkey. J. Neurophysiol. 39, 1069-1080.

457 Freedman, D.J., and Assad, J.A. (2006). Experience-dependent representation of visual

458 categories in parietal cortex. Nature 443, 85-88. 
459 Funahashi, S., Bruce, C.J., and Goldman-Rakic, P.S. (1989). Mnemonic coding of visual space in

460 the monkey's dorsolateral prefrontal cortex. J. Neurophysiol. 61, 331-349.

461 Fuster, J.M. (1973). Unit activity in prefrontal cortex during delayed-response performance:

462 neuronal correlates of transient memory. J. Neurophysiol. 36, 61-78.

463 Fuster, J.M., and Alexander, G.E. (1971). Neuron activity related to short-term memory. Science

$464 \quad 173,652-654$.

465 Giessel, A.J., and Datta, S.R. (2014). Olfactory maps, circuits and computations. Curr. Opin.

466 Neurobiol. 24, 120-132.

467 Gold, J.I., and Shadlen, M.N. (2003). The influence of behavioral context on the representation

468 of a perceptual decision in developing oculomotor commands. J. Neurosci. 23, 632-651.

469 Gold, J.I., and Shadlen, M.N. (2007). The Neural Basis of Decision Making. Annu. Rev.

470 Neurosci. 30, 535-574.

471 Goldman-Rakic, P.S. (1995). Cellular basis of working memory. Neuron 14, 477-485.

472 Guo, Z., Li, N., Huber, D., Ophir, E., Gutnisky, D., Ting, J., Feng, G., and Svoboda, K. (2014).

473 Flow of cortical activity underlying a tactile decision in mice. Neuron 81, 179-194.

474 Guo, Z. V., Inagaki, H.K., Daie, K., Druckmann, S., Gerfen, C.R., and Svoboda, K. (2017).

475 Maintenance of persistent activity in a frontal thalamocortical loop. Nature.

476 Hanks, T.D., Kopec, C.D., Brunton, B.W., Duan, C.A., Erlich, J.C., and Brody, C.D. (2015).

477 Distinct relationships of parietal and prefrontal cortices to evidence accumulation. Nature 520,

$478220-223$.

479 Harvey, C., Coen, P., and Tank, D. (2012). Choice-specific sequences in parietal cortex during a

480 virtual-navigation decision task. Nature.

481 Jones, E.G. (1998). Commentary Viewpoint : the Core and Matrix of Thalamic. Neuroscience 
483 Kadohisa, M., Petrov, P., Stokes, M., Sigala, N., Buckley, M., Gaffan, D., Kusunoki, M., and

484 Duncan, J. (2013). Dynamic Construction of a Coherent Attentional State in a Prefrontal Cell

485 Population. Neuron 80, 235-246.

486 Larkum, M.E., Zhu, J.J., and Sakmann, B. (1999). A new cellular mechanism for coupling inputs

487 arriving at different cortical layers. Nature 398, 338-341.

488 Larkum, M.E., Nevian, T., Sandler, M., Polsky, A., and Schiller, J. (2009). Synaptic integration

489 in tuft dendrites of layer 5 pyramidal neurons: a new unifying principle. Science 325, 756-760.

490 Li, N., Chen, T.-W., Guo, Z. V., Gerfen, C.R., and Svoboda, K. (2015). A motor cortex circuit

491 for motor planning and movement. Nature 519, 51-56.

492 Liu, D., Gu, X., Zhu, J., Zhang, X., Han, Z., Yan, W., Cheng, Q., Hao, J., Fan, H., Hou, R., et al.

493 (2014). Medial prefrontal activity during delay period contributes to learning of a working

494 memory task. Science (80-. ). 346, 458-463.

495 Luo, L., Callaway, E.M., and Svoboda, K. (2018). Genetic Dissection of Neural Circuits: A

496 Decade of Progress. Neuron 98, 256-281.

497 Madisen, L., Garner, A.R., Carandini, M., Zeng, H., Madisen, L., Garner, A.R., Shimaoka, D.,

498 Chuong, A.S., Klapoetke, N.C., Li, L., et al. (2015). Transgenic Mice for Intersectional

499 Targeting of Neural Sensors and Effectors with High Specificity and Performance

500 NeuroResource Transgenic Mice for Intersectional Targeting of Neural Sensors and Effectors

501 with High Specificity and Perf. 942-958.

502 Mainen, Z.F., and Kepecs, A. (2009). Neural representation of behavioral outcomes in the

503 orbitofrontal cortex. Curr. Opin. Neurobiol. 19, 84-91.

504 Mongillo, G., Barak, O., and Tsodyks, M. (2008). Synaptic Theory of Working Memory. 
Science (80-. ). 319, 1543-1546.

Padoa-Schioppa, C., and Assad, J. a (2006). Neurons in the orbitofrontal cortex encode economic

507 value. Nature 441, 223-226.

508 Polsky, A., Mel, B.W., and Schiller, J. (2004). Computational subunits in thin dendrites of

509 pyramidal cells. Nat. Neurosci. 7, 621-627.

510 Ramus, S.J., and Eichenbaum, H. (2000). Neural correlates of olfactory recognition memory in

511 the rat orbitofrontal cortex. J. Neurosci. 20, 8199-8208.

512 Romo, R., Brody, C.D., Hernández, a, and Lemus, L. (1999). Neuronal correlates of parametric

513 working memory in the prefrontal cortex. Nature 399, 470-473.

514 Selen, L.P.J., Shadlen, M.N., and Wolpert, D.M. (2012). Deliberation in the Motor System:

515 Reflex Gains Track Evolving Evidence Leading to a Decision. J. Neurosci. 32, 2276-2286.

516 Shadlen, M.N., and Kiani, R. (2013). Decision making as a window on cognition. Neuron 80,

$517 \quad 791-806$.

518 Shadlen, M.N., and Newsome, W.T. (2001). Neural Basis of a Perceptual Decision in the Parietal

519 Cortex (Area LIP) of the Rhesus Monkey. J. Neurophysiol. 86, 1916-1936.

520 Shadlen, M.N., and Shohamy, D. (2016). Decision Making and Sequential Sampling from

521 Memory. Neuron 90, 927-939.

522 Snyder, L.H., Batista, A.P., and Andersen, R.A. (1997). Coding of intention in the posterior

523 parietal cortex. Nature 386, 167-170.

524 Sosulski, D.L., Bloom, M.L., Cutforth, T., Axel, R., and Datta, S.R. (2011). Distinct

525 representations of olfactory information in different cortical centres. Nature 472, 213-216.

526 Spivey, M.J., Grosjean, M., and Knoblich, G. (2005). From The Cover: Continuous attraction

527 toward phonological competitors. Proc. Natl. Acad. Sci. 102, 10393-10398. 
528 Svoboda, K., and Li, N. (2018). Neural mechanisms of movement planning: motor cortex and

529 beyond. Curr. Opin. Neurobiol. 49, 33-41.

530 Yang, T., and Shadlen, M.N. (2007). Probabilistic reasoning by neurons. Nature 447, 1075-

5311080.

532 Yang, G.R., Murray, J.D., and Wang, X.-J. (2016). A dendritic disinhibitory circuit mechanism

533 for pathway-specific gating. Nat. Commun. 7, 12815.

534 Zhao, S., Ting, J.T., Atallah, H.E., Qiu, L., Tan, J., Gloss, B., Augustine, G.J., Deisseroth, K.,

535 Luo, M., Graybiel, A.M., et al. (2011). Cell type-specific channelrhodopsin-2 transgenic mice

536 for optogenetic dissection of neural circuitry function. Nat. Methods 8, 745-752.

537 Zingg, B., Hintiryan, H., Gou, L., Song, M.Y., Bay, M., Bienkowski, M.S., Foster, N.N.,

538 Yamashita, S., Bowman, I., Toga, A.W., et al. (2014). Neural networks of the mouse neocortex.

539 Cell 156, 1096-1111. 


\section{Acknowledgements}

544 We thank T. Harris for the acute H3 probes; T. Tabachnik, D. Peterka, G. Johnson, J. Wang, J.

545 Goldbas, A. Baker and M. Chin for technic assistance. K. Svoboda, Z. Guo, L. Abbott, A.

546 Losonczy, R. Bruno, C. Rodgers, C. Lacefield, K. Shin, and B. Hu for advice on experiments and

547 analysis; D. Wolpert and members of the Axel and Shadlen labs for helpful discussions; C. Eccard

548 for assistance in the preparation of the manuscript; M. Myles for figure artwork. Z.W. is a Simons

549 Junior Fellow. A.L.-K. is supported by NSF NeuroNex Award DBI-1707398, the Burroughs

550 Wellcome Foundation, and the Gatsby Charitable Foundation. R.A. and M.N.S. are supported by

551 the Howard Hughes Medical Institute (HHMI). R.A. is also supported by the Simons Foundation.

\section{Author Contributions}

554 Z.W., R.A. and M.N.S. conceived and designed the study. Z.W. performed all the experiments 555 with assistance from P.S. and A.T. A.L.-K. implemented the attractor network models. Z.W., A.L.556 K., R.A. and M.N.S. interpreted the results and wrote the paper.

\section{Competing interests}

559 The authors declare no competing financial interests.

\section{Data and materials availability}

562 The data, code, and materials that support the findings of this study are available from the 563 corresponding authors upon reasonable request. 


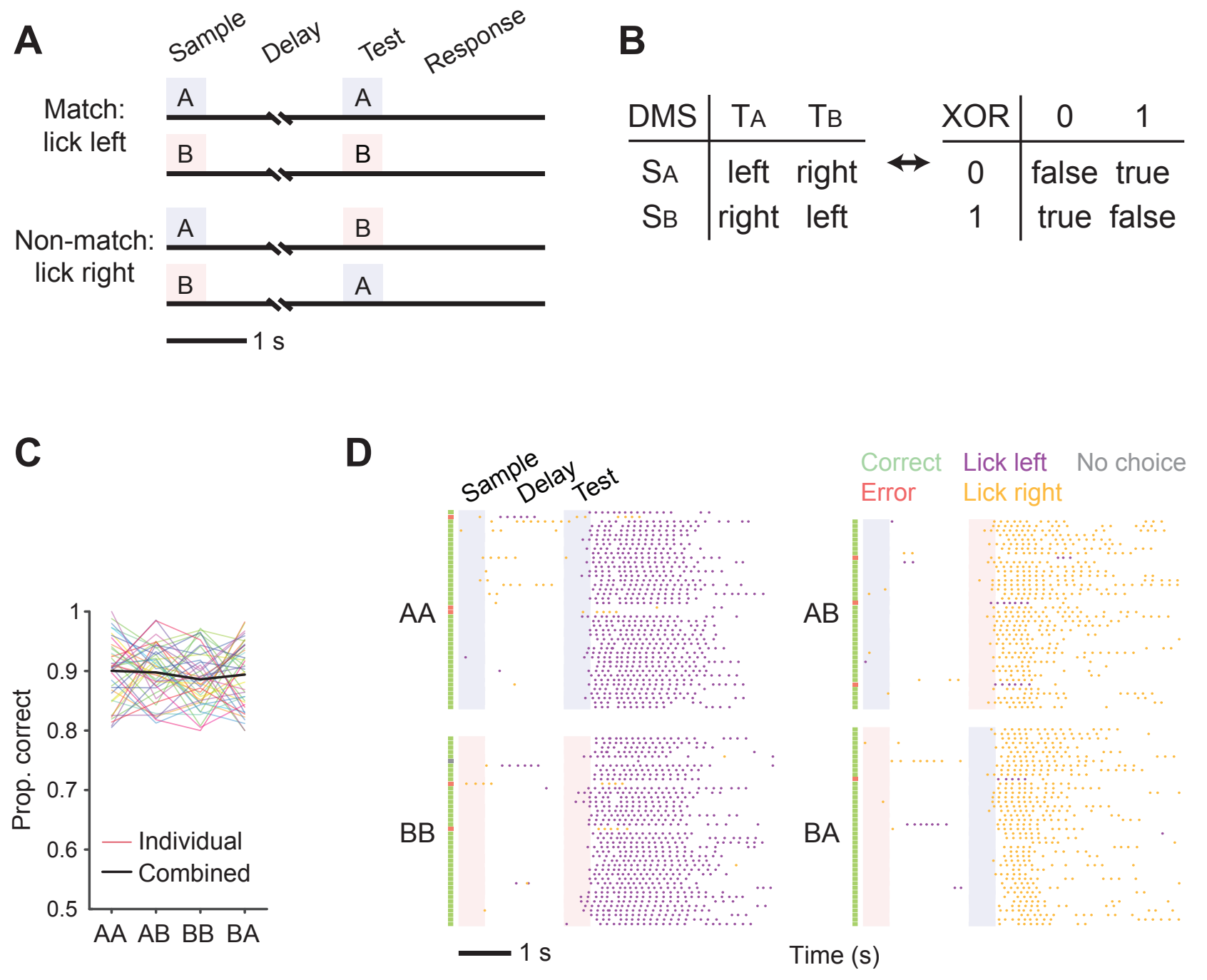




\section{Figure 1: Lick-left/lick-right olfactory delayed match to sample task.}

567 (A) Task structure. Mice are presented with two odorants (A: pinene, B: hexenol) separated by a

568 delay and must decide whether they are the same (match) or different (non-match). The two odors

569 create four unique pairs, or trial types. Match trials are rewarded on the left port and non-match on

570 the right (see Methods).

571 (B) The DMS task is a variant of the logical XOR problem. The outcome/response depends on

572 whether the two bits of information are the same or not.

573 (C) The proportion of correct trials in well-trained animals. Each colored line represents data from

574 one animal and the black line is the mean of all animals $(n=41)$.

575 (D) An example behavior session from a well-trained animal. The four trial types are randomly

576 presented in the session but grouped here for plotting. Each row is a trial. The green, red, and gray

577 markers on the left side of each trial denote respectively the outcome of correct, error, and no

578 choice. The A and B odors are indicated by the light blue and light red shadings. The magenta and

579 yellow tick marks are the left and right licks, respectively. Mice were trained to suppress their

580 premature licks during the sample and delay epochs. The sample and test epochs are $0.5 \mathrm{~s}$ each

581 and the delay is $1.5 \mathrm{~s}$.

582 See also Figure S1. 
bioRxiv preprint doi: https://doi.org/10.1101/757104; this version posted September 3, 2019. The copyright holder for this preprint (which was not certified by peer review) is the author/funder, who has granted bioRxiv a license to display the preprint in perpetuity. It is made available

Figure 2 under aCC-BY-NC-ND 4.0 International license.
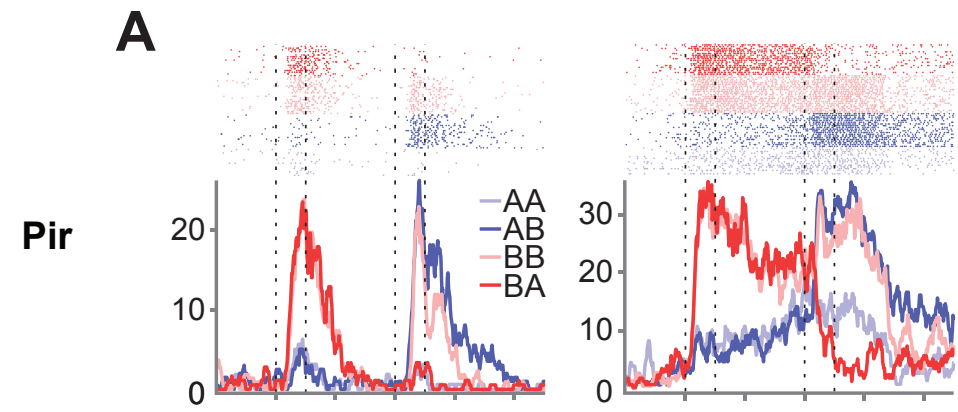

B
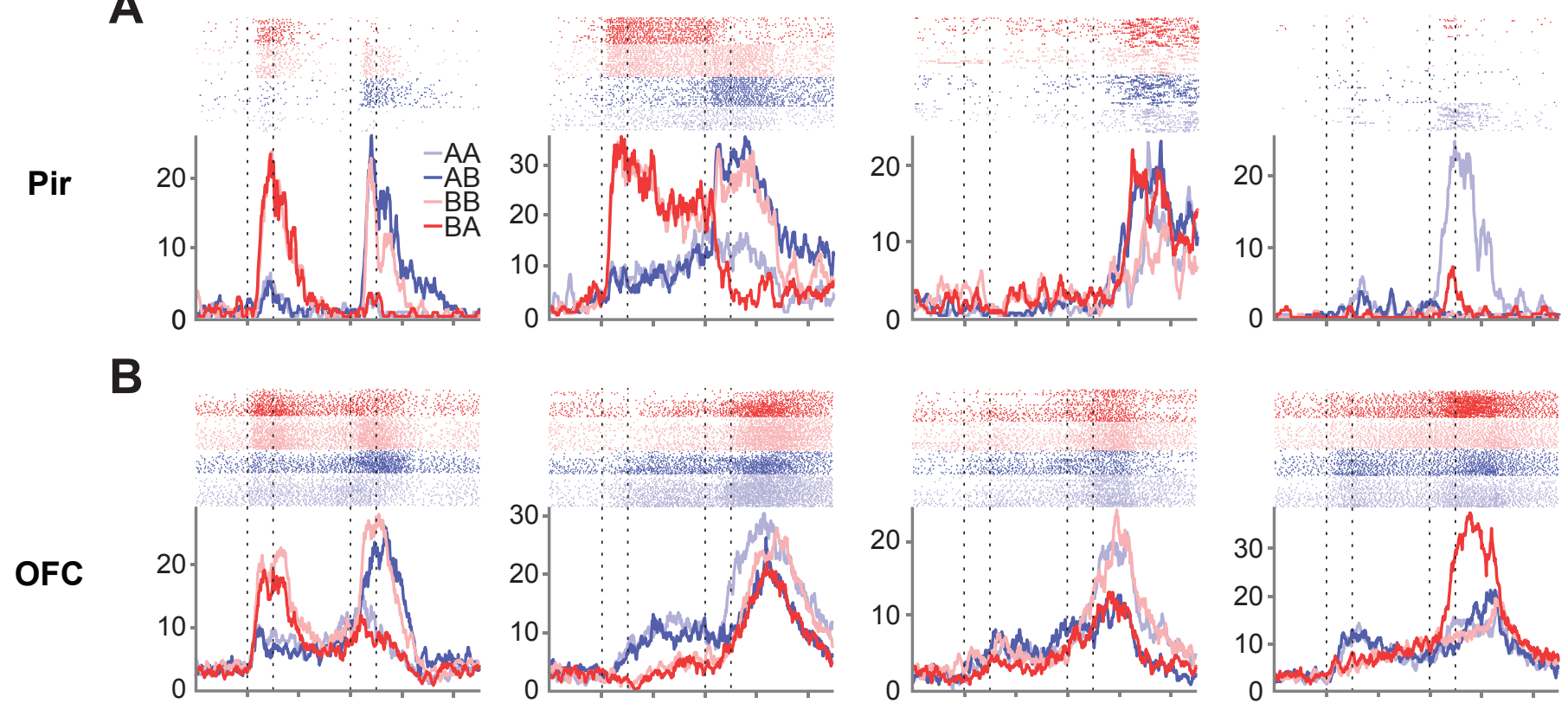

C

ALM
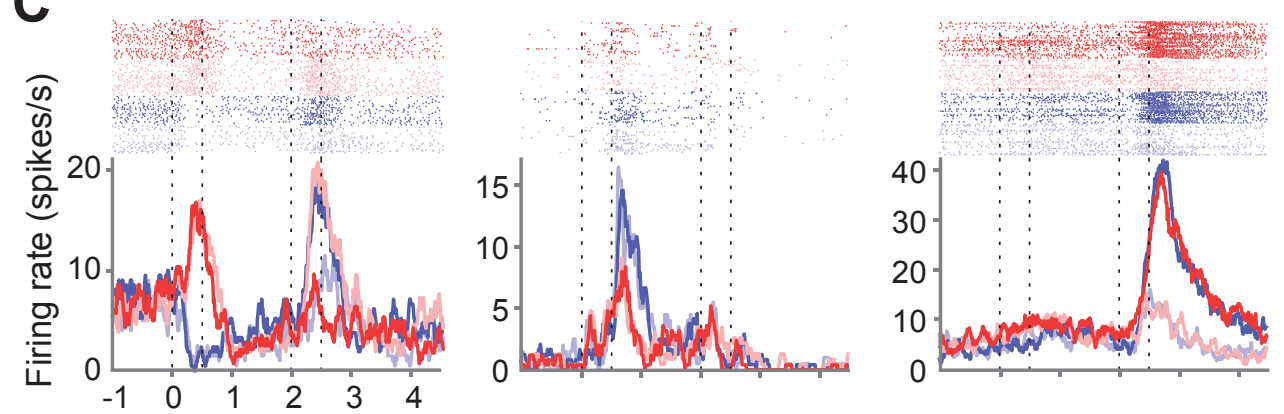

Time from sample onset (s)

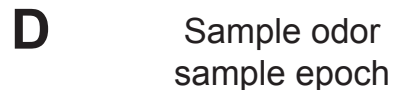

Pir
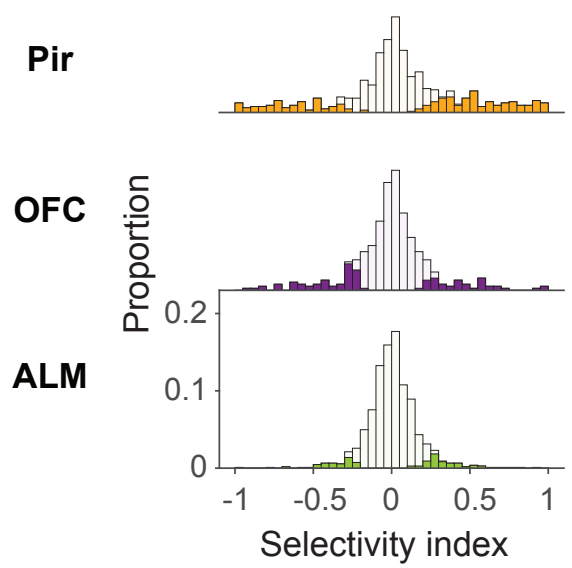

F Test odor
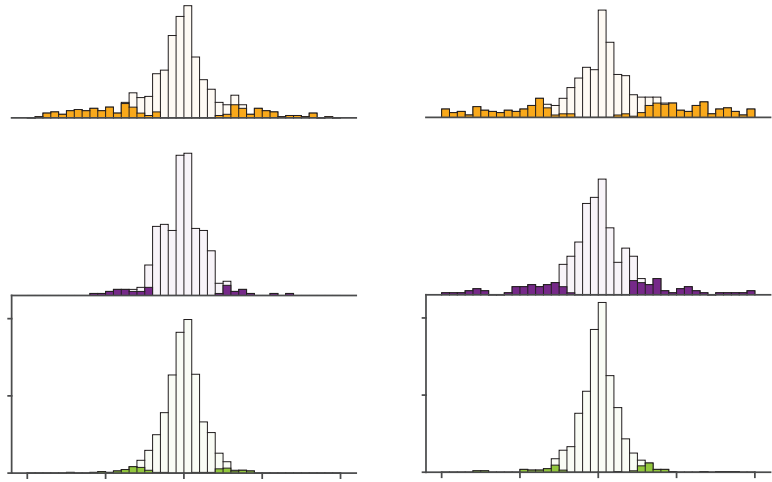
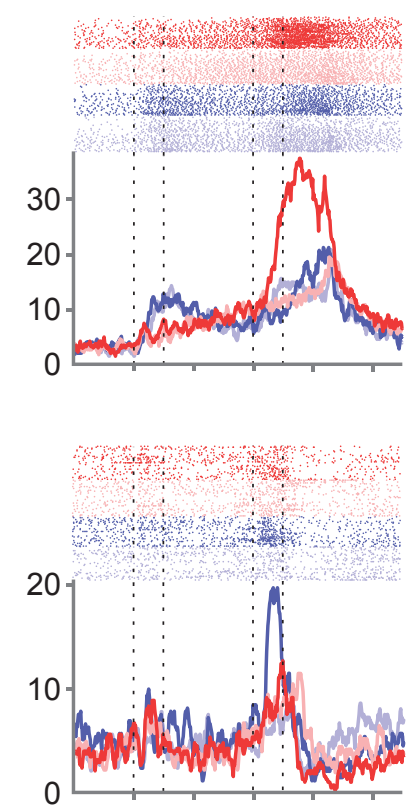

G

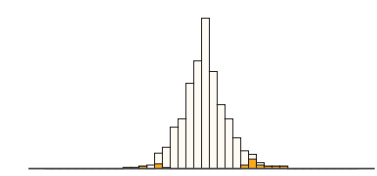

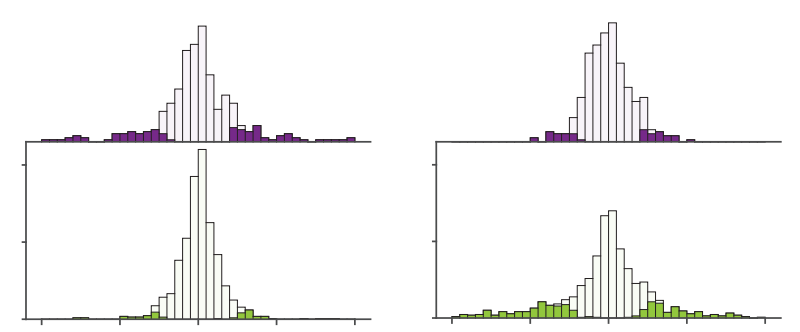




\section{Figure 2: Neural recordings of Pir, OFC and ALM.}

585 (A-C) Firing rates of example neurons from Pir (A), OFC (B), and ALM (C) recordings. The

586 rasters (top) show the times of action potentials in individual trials (rows). Traces are mean firing

587 rates (100 ms bins) across trials. The dotted lines indicate the sample and test epochs hereafter.

588 (D-G) Selectivity indices of Pir, OFC, and ALM neurons for sample odor during the sample epoch

589 (D), sample odor during the last $0.5 \mathrm{~s}$ of the delay epoch (E), test odor during the test epoch (F),

590 and choice during the test epoch $(\mathrm{G}) . \mathrm{n}=647,380$ and 1086 neurons for Pir, OFC and ALM,

591 respectively. Shading indicates neurons that are selective for an odor (D-F) or behavioral choice

592 (G) determined by Mann-Whitney U test, $P<0.01$, not corrected for multiple comparisons.

593 See also Figure S2.

594

595 
bioRxiv preprint doi: https://doi.org/10 1101/757104 this version posted September 3,2019 . The copyright holder for this preprint (which was not certified by peer review) is the author/funder, who has granted bioRxiv a license to display the preprint in perpetuity. It is made available

\section{Figure 3}

A

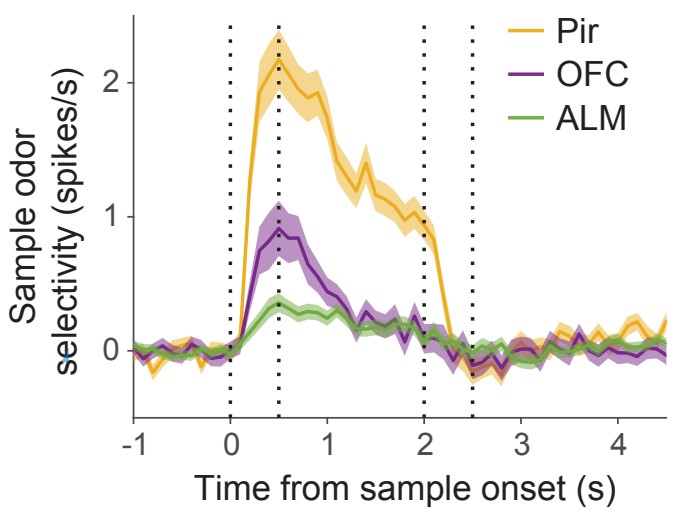

B

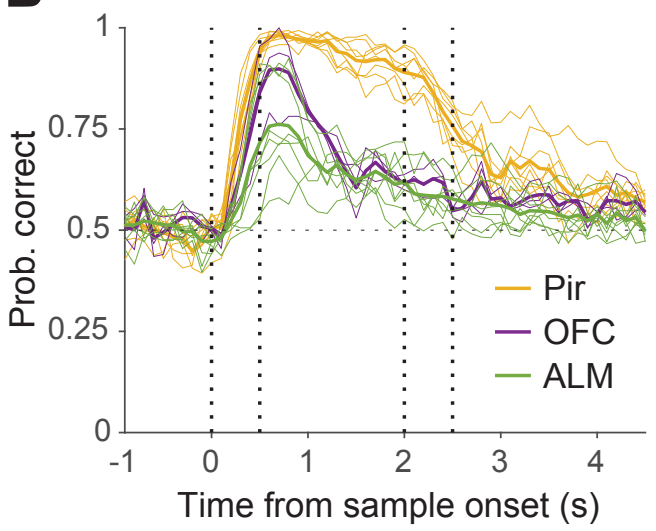

All neurons

Time from sample onset (s) under aCC-BY-NC-ND 4.0 International license.

\section{C}

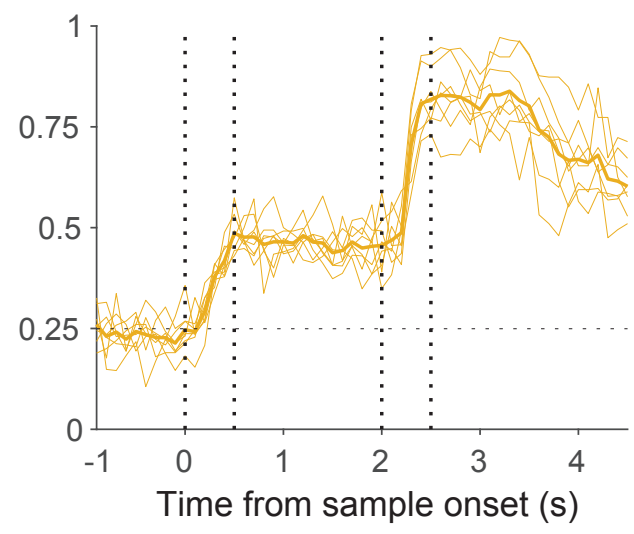

Selective neurons

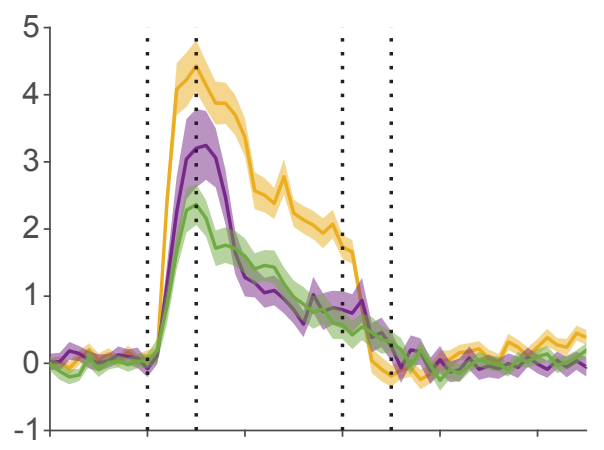

D

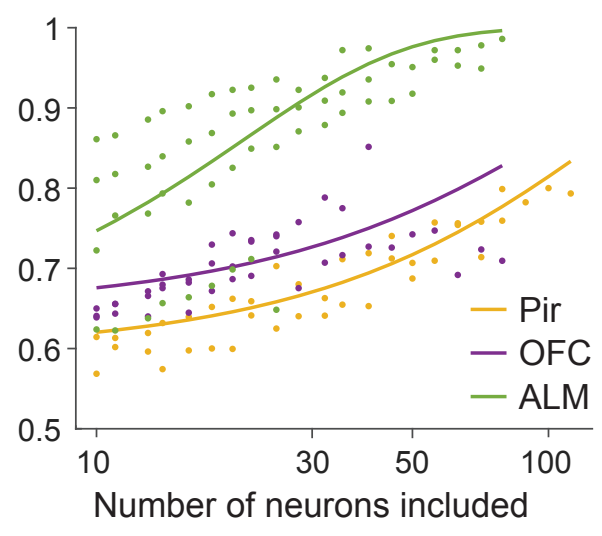




\section{Figure 3: Odor selectivity and decoding task-relevant features using the neural responses.}

597 (A) Population sample odor selectivity of Pir, OFC, and ALM neurons. The ordinate is the

598 difference in firing rate to the preferred and the nonpreferred odor (Methods). The lines represent

599 the means across neurons and the shading represents the s.e.m. Left graph, all recorded neurons

600 from each area contribute to the averages. Right graph, only neurons that are selective for sample

601 odor in the sample and delay epochs contribute to the averages $(n=319,105$ and 147 neurons for

602 Pir, OFC and ALM, respectively).

603 (B) Performance of a support vector machine trained to classify the sample odor identity using

604 simultaneously recorded neurons from each of the three areas. Each thin line represents the

605 probability of correct classifications of the "held out" trials using data from one session (see

606 Methods). Thick lines represent the mean of all sessions from an area (color).

607 (C) Performance of a classifier of trial type using all simultaneously recorded Pir neurons from 608 each session. Same conventions as in (A).

609 (D) Performance of a classifier of match/non-match trials using the neural responses after test odor

610 onset and before animal's first lick. Each point represents the performance using $N$ randomly

611 selected neurons as input to the classifier, calculated using sessions in which at least $N$ neurons

612 were recorded simultaneously. The traces are fits to the classifier performance (logistic regression).

613 For each area, the performance improves as more neurons are included. 
bioRxiv preprint doi: https://doi.org/10.1101/757104; this version posted September 3, 2019. The copyright holder for this preprint (which was Fig Dpregrtified by peer review) is the author/funder, who has granted bioRxiv a license to display the preprint in perpetuity. It is made available

A

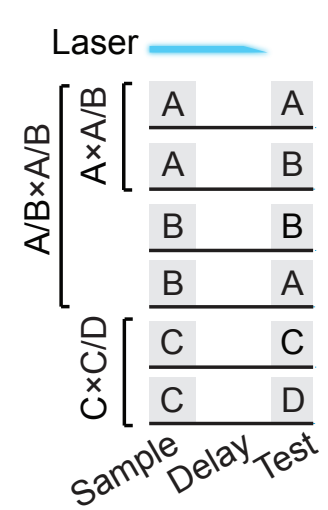

F $\quad A \times A / B$ control

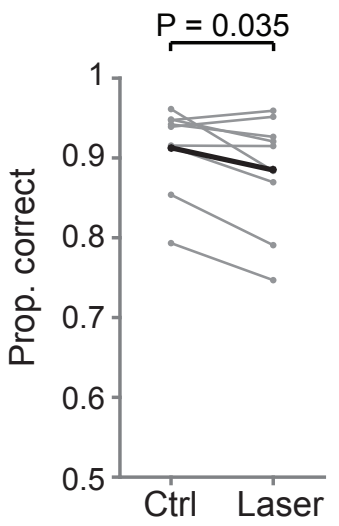

B ALM inhibition
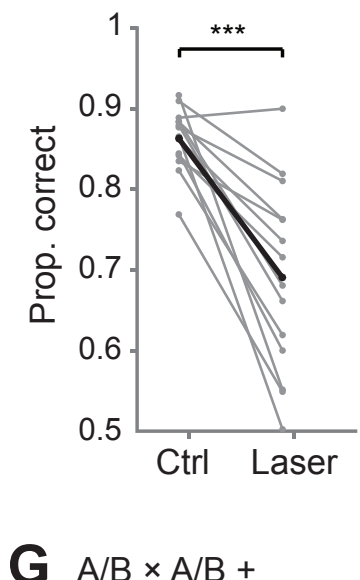
$C \times C / D$ control

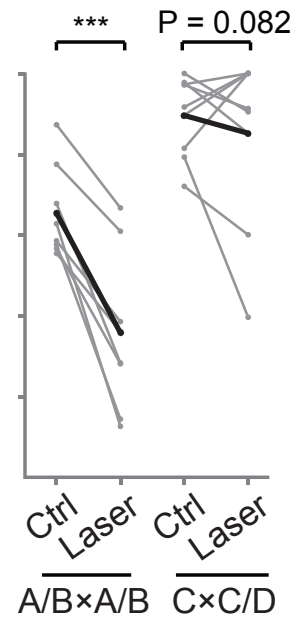

C OFC inhibition

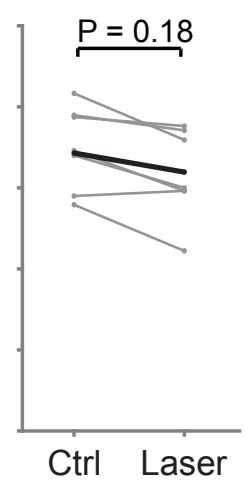

H
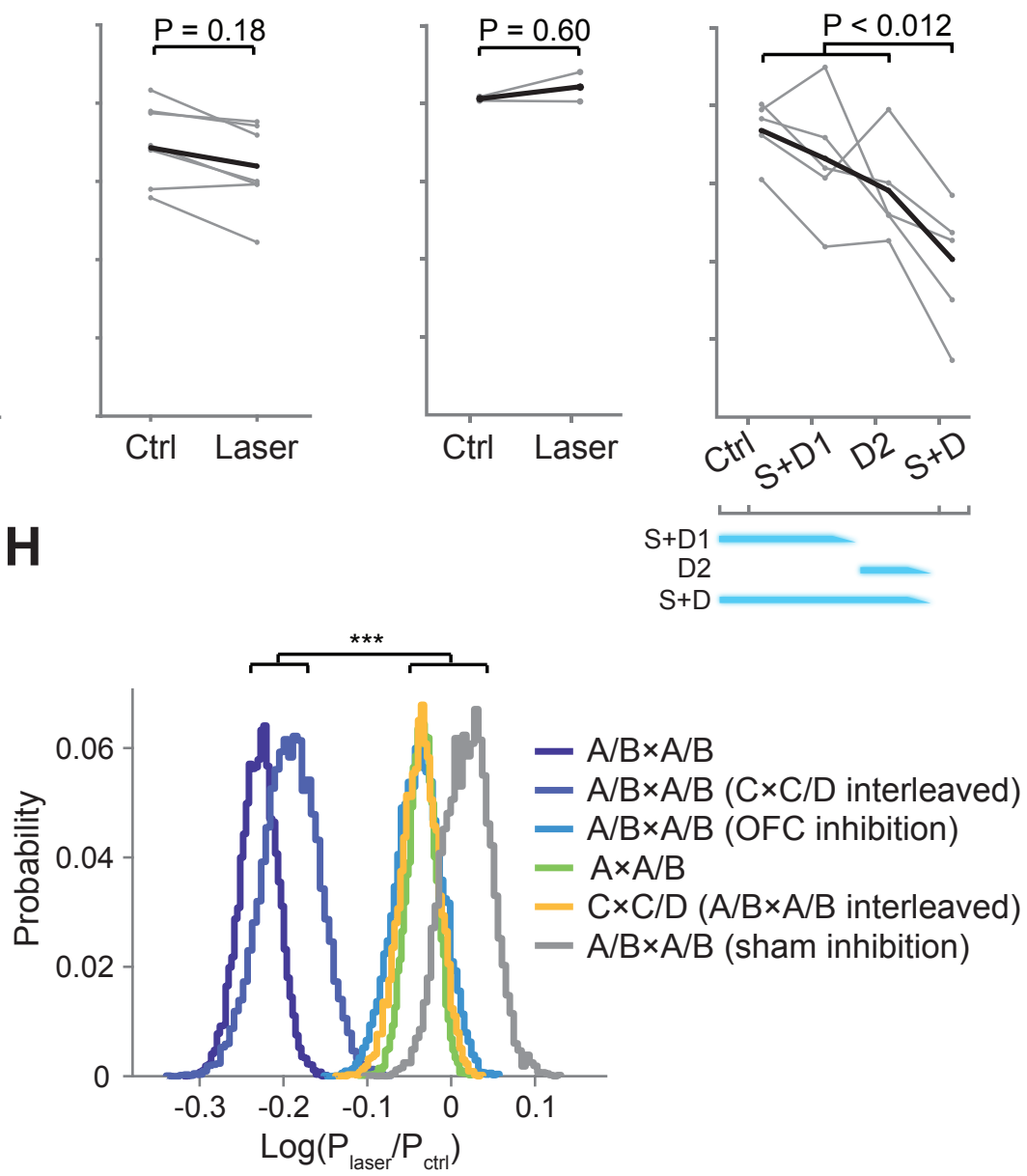

E S+D breakdown

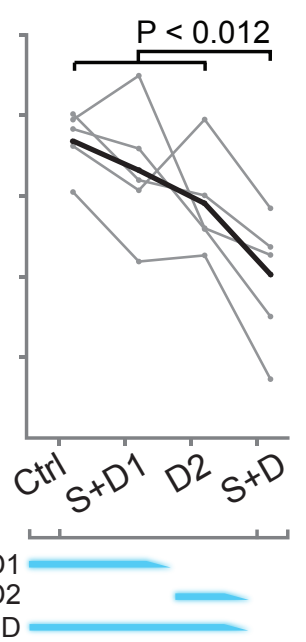

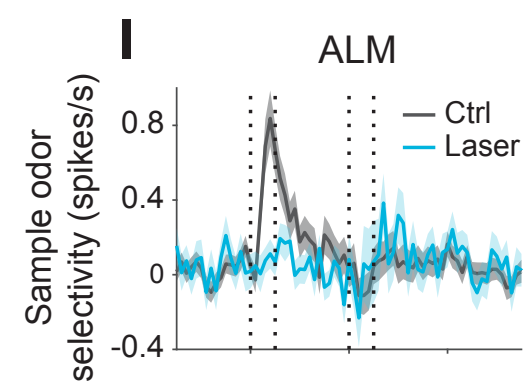
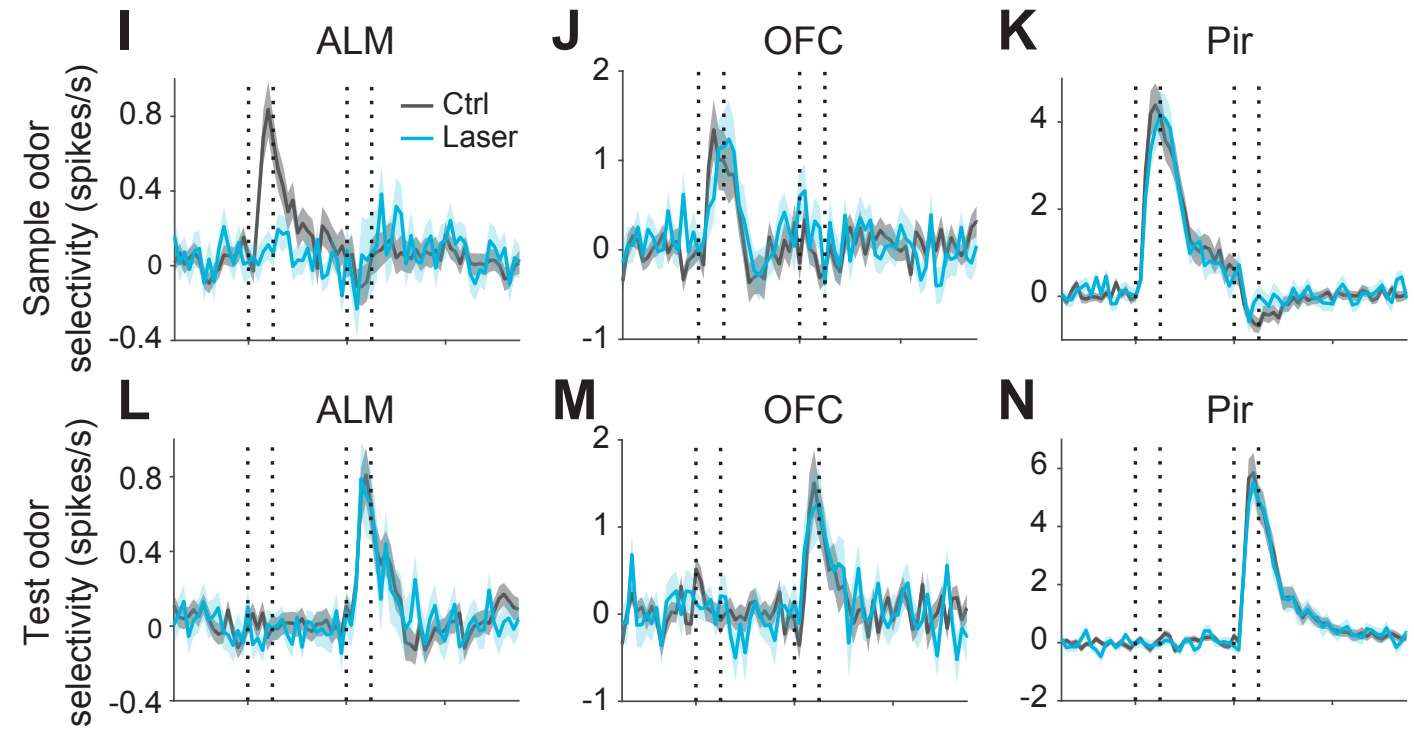
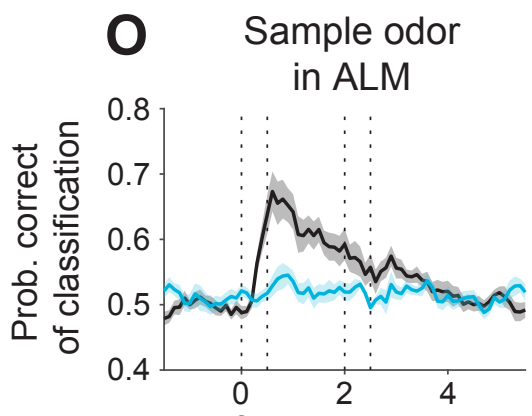

Time from sample onset (s)
$\mathbf{P}$

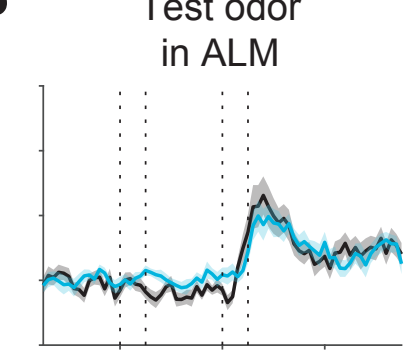

Q Match/non-match

in Pir

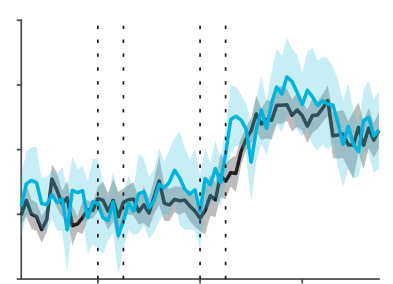

R Match/non-match in OFC

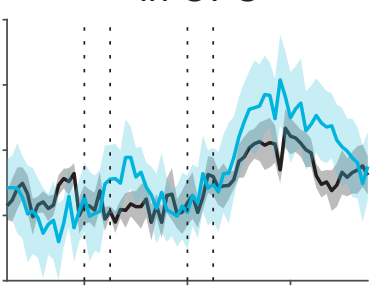


Figure 4: Optogenetic inactivation experiments.

617 (A) Schematic of the DMS and control tasks employed in the inhibition experiments (time not 618 drawn to scale). $\mathrm{A} / \mathrm{B} \times \mathrm{A} / \mathrm{B}$ design is the same DMS task as in the recording experiments. On 619 inactivation trials, unless otherwise indicated, the laser was on for $2 \mathrm{~s}$ including a $0.25 \mathrm{~s}$ power 620 ramp-down at the end (tapered blue bar). In the interleaved $\mathrm{A} / \mathrm{B} \times \mathrm{A} / \mathrm{B} \& \mathrm{C} \times \mathrm{C} / \mathrm{D}$ task, the delay 621 epoch is $4 \mathrm{~s}$ (Methods).

622 (B) Proportion of correct trials in the $\mathrm{A} / \mathrm{B} \times \mathrm{A} / \mathrm{B}$ task with and without bilateral ALM inactivation.

623 Each thin line represents data from one animal. The thick line is the combined performance from 6244092 trials (14 animals). Statistical reliability of effects in panels (B-G) are based on permutation 625 tests $(*, * *$ and $* * *$ denote $P<0.01,0.001$ and 0.0001$)$.

626 (C) Proportion of correct trials in the $\mathrm{A} / \mathrm{B} \times \mathrm{A} / \mathrm{B}$ task with and without bilateral OFC inactivation 627 (7 animals, 2278 trials).

628 (D) Proportion of correct trials in the $\mathrm{A} / \mathrm{B} \times \mathrm{A} / \mathrm{B}$ task by $\mathrm{ChR} 2$ non-carriers with and without sham 629 ALM inactivation (2 animals, 437 trials).

630 (E) Effect of inactivation during portions of the sample and delay epochs (5 animals, 3732 trials).

631 Same conventions as in (B-D). Ctrl, no inactivation; $S+D 1$, sample and first 1.5 s of the delay; $D 2$,

632 last 1.0 s of the delay; $S+D$, sample and delay.

633 (F) Proportion of correct trials in the $\mathrm{A} \times \mathrm{A} / \mathrm{B}$ control task with and without bilateral ALM bilateral 634 inactivation (9 animals, 2198 trials).

635 (G) Proportion of correct trials in the interleaved $\mathrm{A} / \mathrm{B} \times \mathrm{A} / \mathrm{B} \& \mathrm{C} \times \mathrm{C} / \mathrm{D}$ task with and without bilateral ALM inactivation (9 animals, 2728 trials).

637 (H) ALM inactivation caused greater impairment on the $\mathrm{A} / \mathrm{B} \times \mathrm{A} / \mathrm{B}$ task than on the control tasks 638 and $\mathrm{OFC}$ inactivation on the same $\mathrm{A} / \mathrm{B} \times \mathrm{A} / \mathrm{B}$ task. The effect of inactivation is expressed as the 
$639 \log$ odds (LO) of the mouse performance with and without inactivation (Methods, Eq. 4).

640 Distributions of the LO were estimated by a bootstrap procedure. The largest effects were evident

641 when ALM was inactivated and the sample was informative ( $t$-test, $d . f$. determined by size of data

642 set).

643 (I-K) Population sample odor selectivity of ALM, OFC, and Pir with and without ALM

644 inactivation. The ordinate is the difference in firing rate to the preferred and the nonpreferred odor

645 across neurons (see Methods). Note the different scales. Shading is s.e.m. $n=470,104$ and 188

646 neurons for ALM, OFC and Pir, respectively. The same neurons are used in the analyses below.

647 (L-N), Population test odor selectivity of ALM, OFC, and Pir with and without ALM inactivation.

648 (O-R) Performance of the SVM decoder to classify sample odor identity using ALM responses

649 (O), test odor identity using ALM responses (P), match/non-match using Pir response (Q), and 650 match/non-match using OFC responses (R) with and without ALM inactivation.

651 See also Figure S3 and S4.

652 

Figure 5 under aCC-BY-NC-ND 4.0 International license.

A

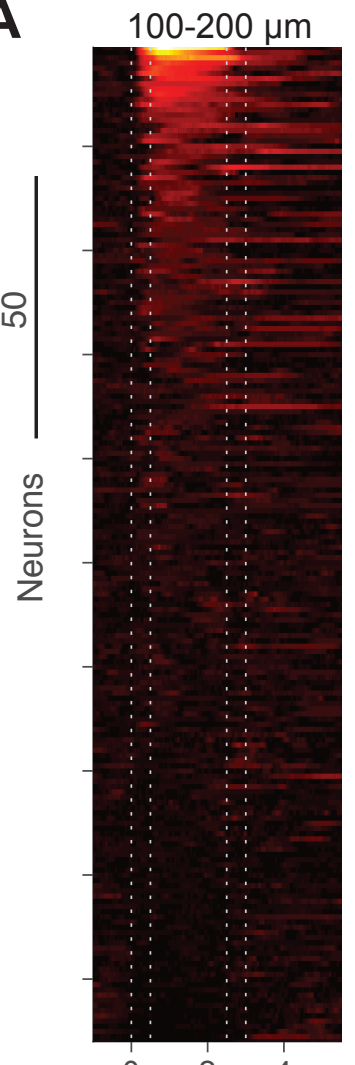

$\begin{array}{lll}0 & 2 & 4\end{array}$

Time from sample onset (s)

B

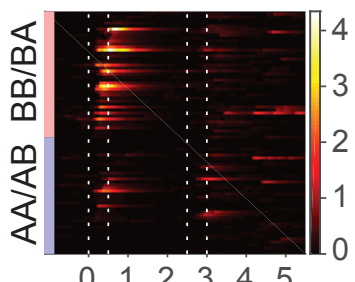

Time from sample onset (s)

\section{C}
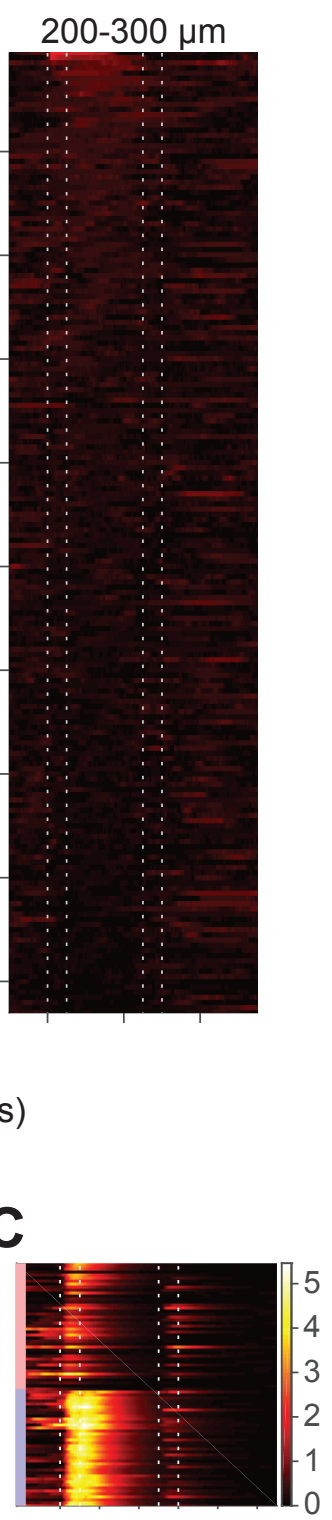

$300-400 \mu \mathrm{m}$

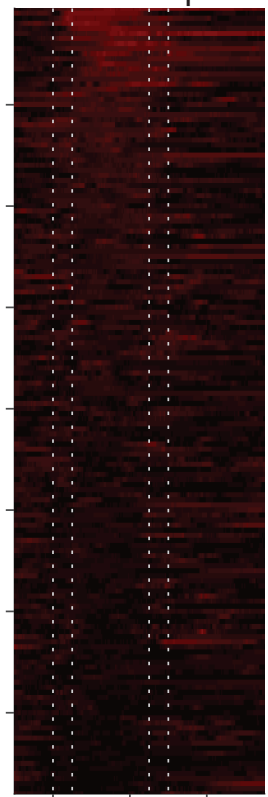

Standardized selectivity

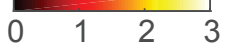

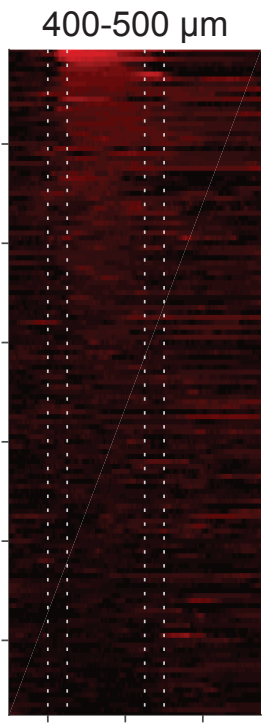
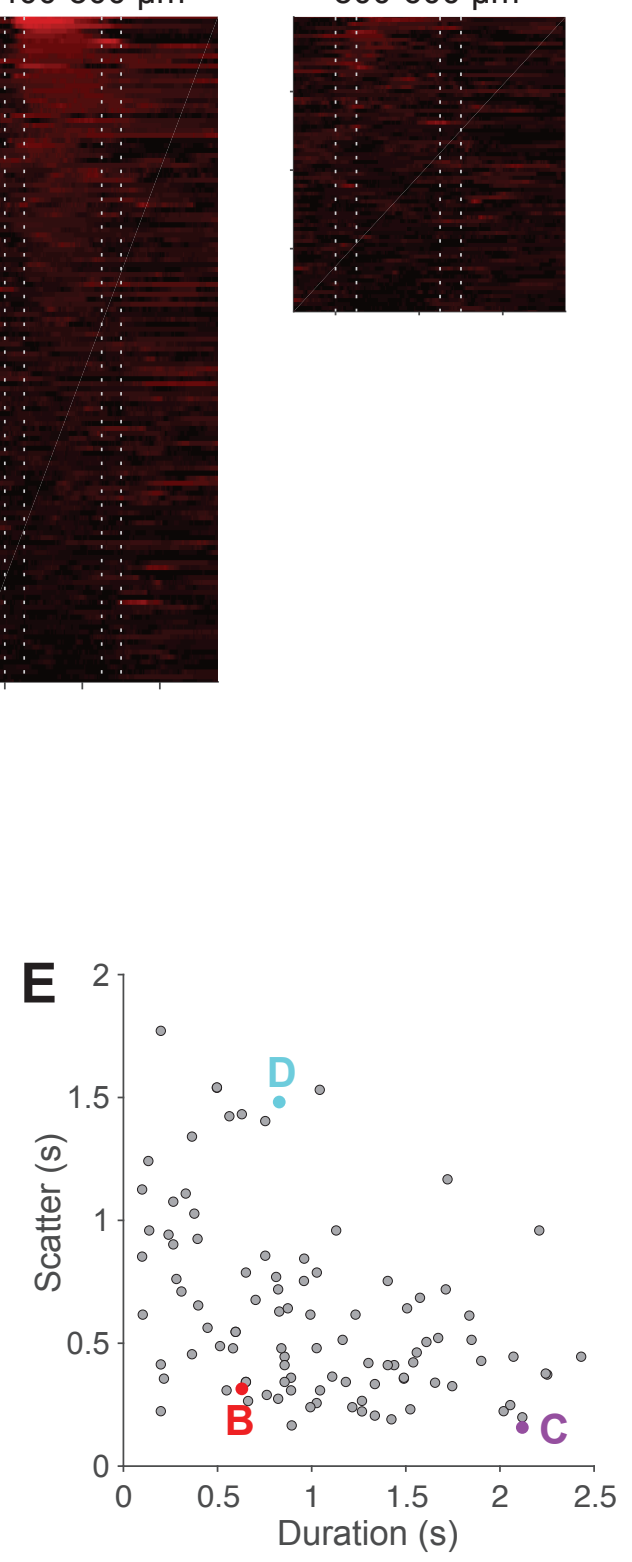

H Choice

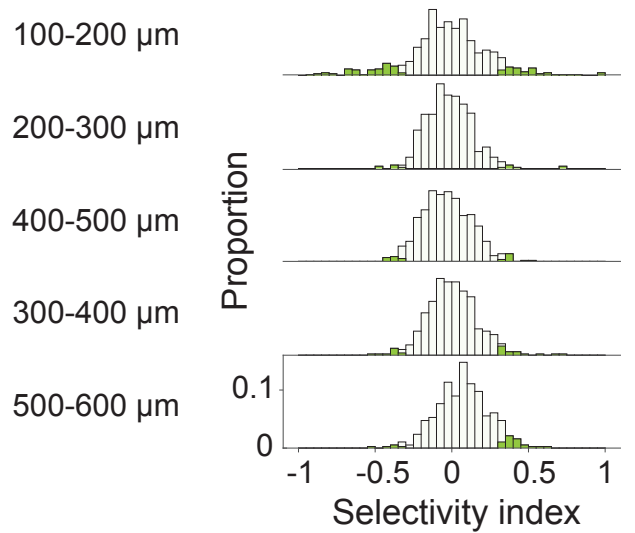

G Test odor
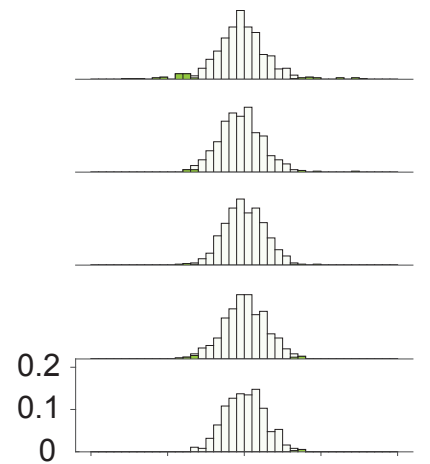
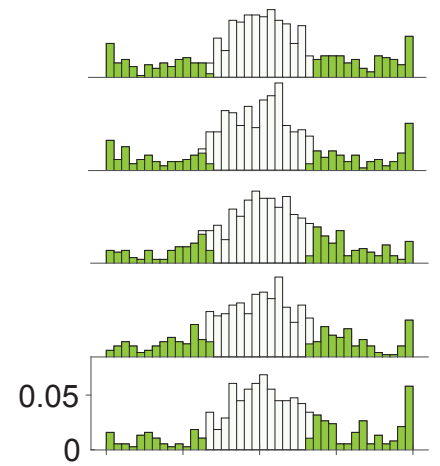


\section{Figure 5: Two-photon calcium imaging of ALM.}

655 (A) Standardized sample odor selectivity of ALM neurons across five different cortical depths in

656 an example animal. The standardized odor selectivity is the difference between the $\Delta \mathrm{F} / \mathrm{F}$ to odor

657 A and B, normalized by the combined standard deviation (Methods). Each row is a neuron, sorted

658 by the mean standardized selectivity during the sample and delay epochs.

659 (B-D) $\Delta \mathrm{F} / \mathrm{F}$ traces of example sample-selective neurons. Each row is a trial, grouped by sample 660 odor identity and sorted by peak response time to sample odor.

661 (E) Time course of sample-selective responses. Each point shows the duration of the calcium 662 transient (abscissa) against the scatter of the peak response (ordinate). Response duration is the 663 median duration of each neuron's response. Scatter is defined as the interquartile range of the peak 664 response times across trials (Methods). The three colored points correspond to the neurons shown 665 in panels (B-D).

666 (F-H) Selectivity indices of neurons across five cortical depths for sample odor, test odor, and 667 choice, respectively. $\mathrm{n}=614,514,608,512$ and 379 neurons for the five cortical depths, 668 respectively, from superficial to deep layers. Shading denotes statistical significance $(P<0.01$, 669 Mann-Whitney U test, two-tailed).

670 See also Figure S5 and S6. 
bioRxiv preprint doi: https://doi.org/10.1101/757104; this version posted September 3, 2019. The copyright holder for this preprint (which was not certified by peer review) is the author/funder, who has granted bioRxiv a license to display the preprint in perpetuity. It is made available Figure 6 under aCC-BY-NC-ND 4.0 International license.

A

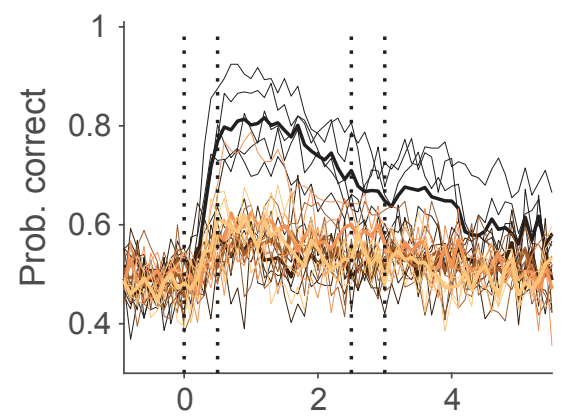

Time from sample onset (s)
B

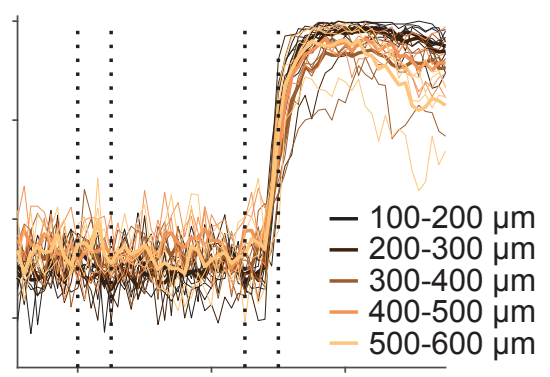

C - Sample odor selective

- Selective for both

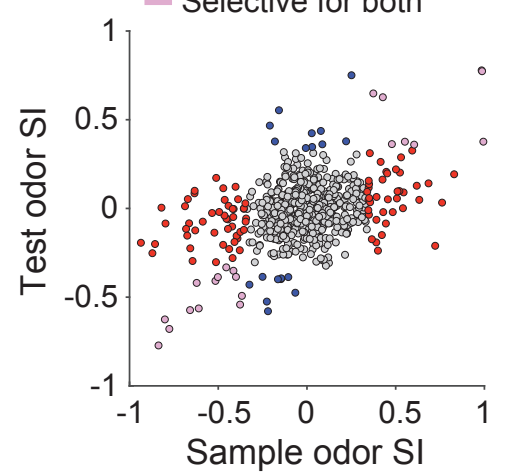

E

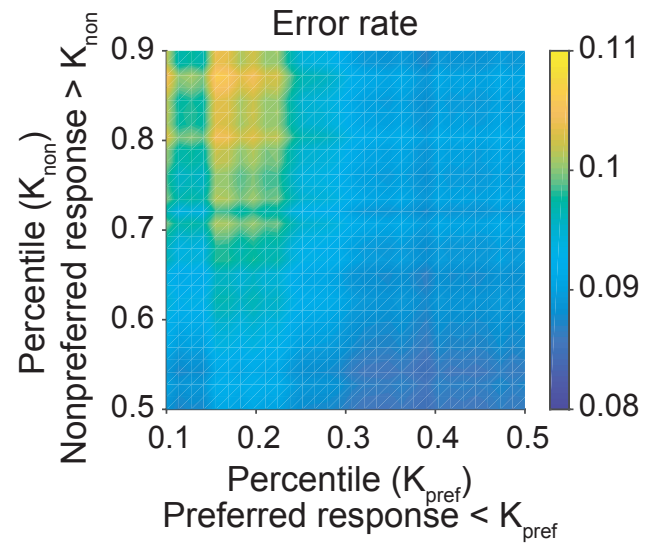

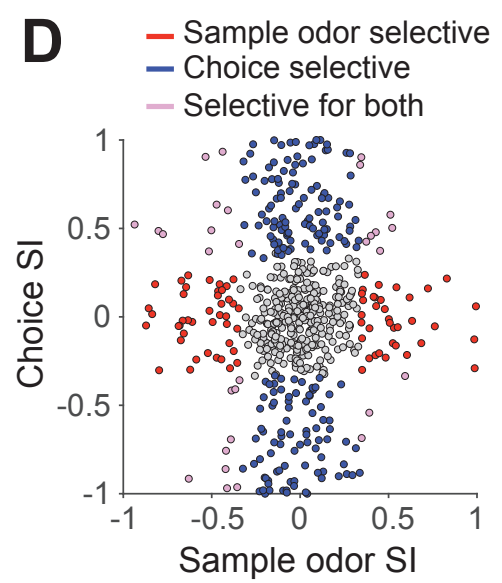




\section{Figure 6: Sample-selective cells in ALM L2.}

$674(\mathrm{~A}, \mathrm{~B})$ Performance of a support vector machine trained to classify the sample odor identity (A) or

675 choice (B) using a subset of simultaneously imaged neurons from each of the five cortical depths.

676 Each thin line represents the probability of correct classifications of the "held out" trials using data

677 from one session (see Methods). Thick lines represent the mean of all sessions from a depth.

678 (C) Selectivity indices of ALM L2 neurons for sample and test odors. Red, blue or purple denotes 679 neurons selective for the sample odor, test odor or both, respectively. Non-selective neurons are 680 shown in gray.

681 (D) Selectivity indices of ALM L2 neurons for sample odor and choice. Red, blue or purple denotes 682 neurons selective for the sample odor, choice or both, respectively. Non-selective neurons are 683 shown in gray.

684 (E) Trial-by-trial association between response and choice accuracy. The heatmap shows the 685 fraction of errors when L2 neurons responded weakly to the preferred odor (pref) or strongly to 686 the nonpreferred odor (non). The criteria for weak and strong are varied parametrically up to the 687 median for pref (abscissa) and above the median for non (ordinate). The graph shows an increased 688 probability of an error when the pref response is in the lower $20^{\text {th }}$ percentile or nonpref response 689 is in the upper $30^{\text {th }}$ percentile. Logistic regression demonstrates that the effect is reliable across the 690 dataset $(P<0.001$; Methods $)$. 
bioRxiv preprint doi: https://doi org/10 1101/757104 - this version posted September 3, 2019. The copyright holder for this preprint (which was not certified by peer review) is the author/funder, who has granted bioRxiv a license to display the preprint in perpetuity. It is made available Figure 7 under aCC-BY-NC-ND 4.0 International license.

A

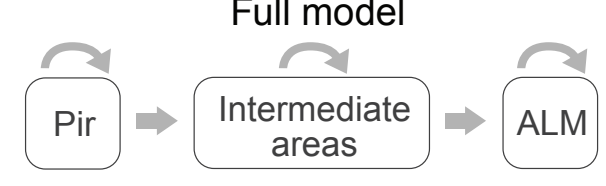

No ALM persistence

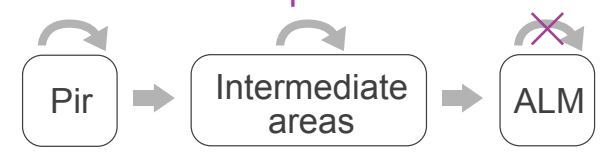

Only ALM persistence

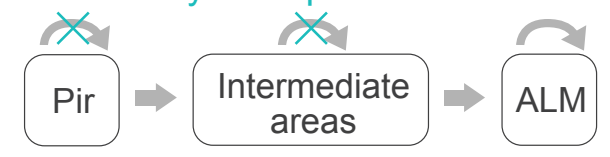

C

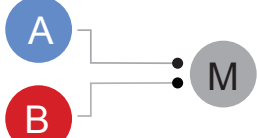

Depression

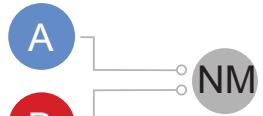

B

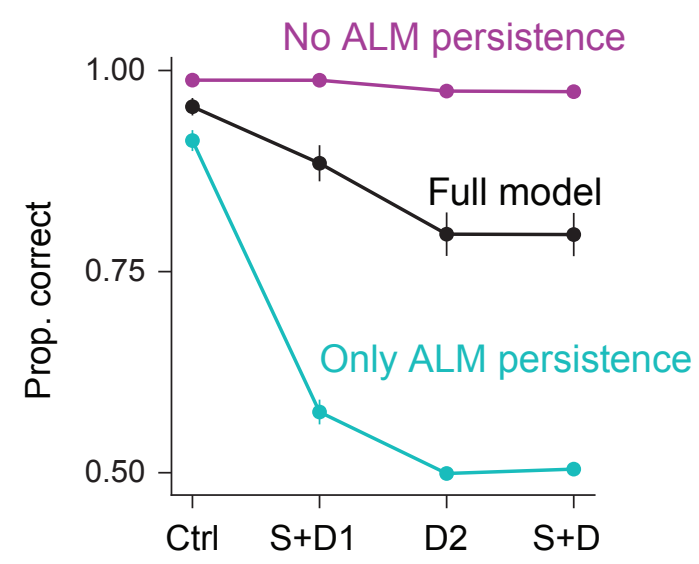

D
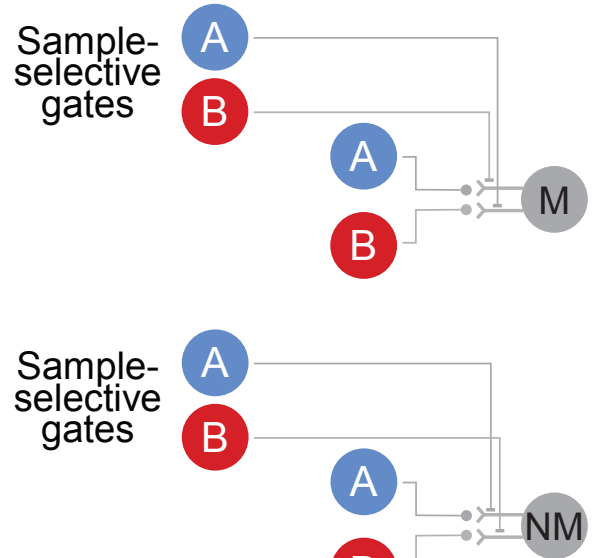

B 


\section{$693 \quad$ Figure 7: Neural models of the DMS task.}

694 (A) Three variants of the recurrent neural network model. Each contains three areas connected by

695 fixed, random, feed forward projections (horizontal straight arrows). Top: all recurrent connections

696 within each layer are trainable. Middle: recurrent connections within Pir and OFC, but not ALM,

697 are trainable. Bottom: only recurrent connections within ALM are trainable. Note that recurrence

698 within an area is necessary to achieve persistent activity that is not inherited from an upstream area.

699 (B) Simulated effects of inactivating ALM during portions of the sample and delay epochs. Same

700 conventions for the behavioral epochs as in Fig. 4E.

701 (C) Synaptic facilitation and depression model. Facilitation leads to enhanced response to repeated

702 presentation of the same stimulus in match selective neurons, whereas depression results in

703 suppression in non-match selective neurons. We assume that the soma is inhibited during the

704 sample epoch so that action potentials only occur after the test odor.

705 (D) Dendritic gating model. Top: circuit supporting match (M). The sample odor suppresses

706 excitatory inputs that convey the identity of the opposite test odor. Bottom: circuit supporting

707 nonmatch (NM). A similar result could be achieved with presynaptic gating. This circuit also

708 requires a mechanism to suppress spiking before the test epoch and a mechanism to reset the gate.

709

710

711 
bioRxiv preprint doi: https://doi.org/10.1101/757104; this version posted September 3, 2019. The copyright holder for this preprint (which was not certified by peer review) is the author/funder, who has granted bioRxiv a license to display the preprint in perpetuity. It is made available Figure S1

A

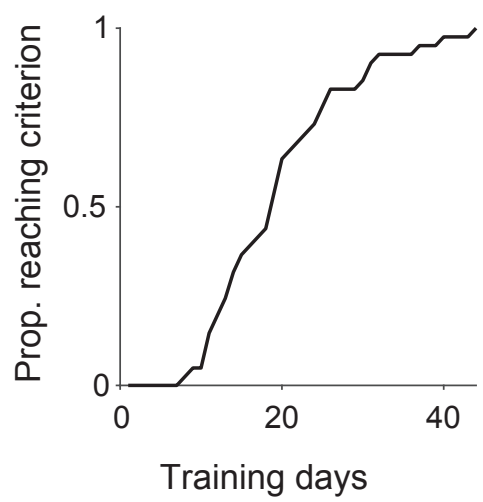

C

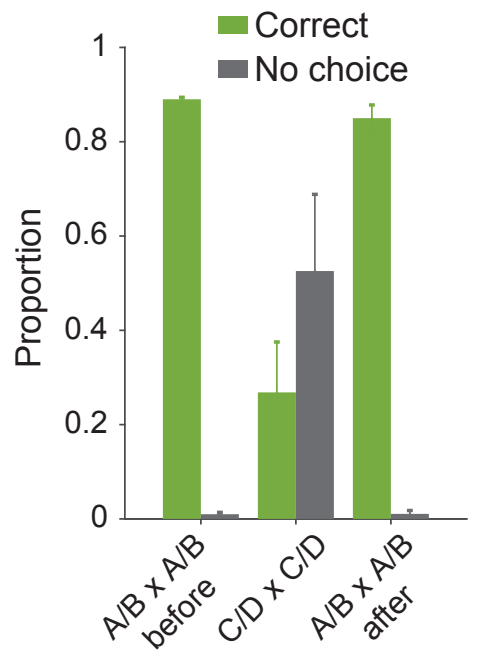

E

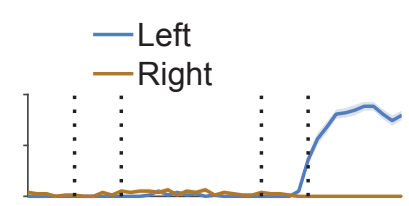

AA

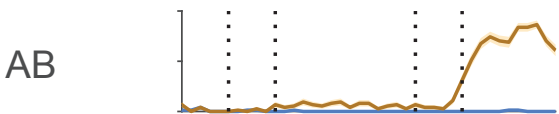

$\mathrm{BB}$

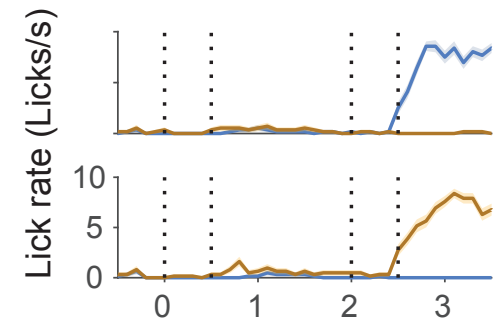

Time from sample onset (s)
B

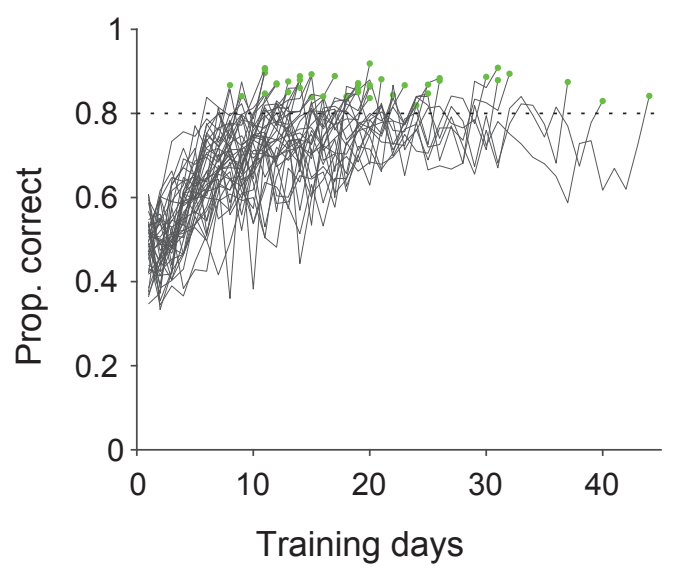

D
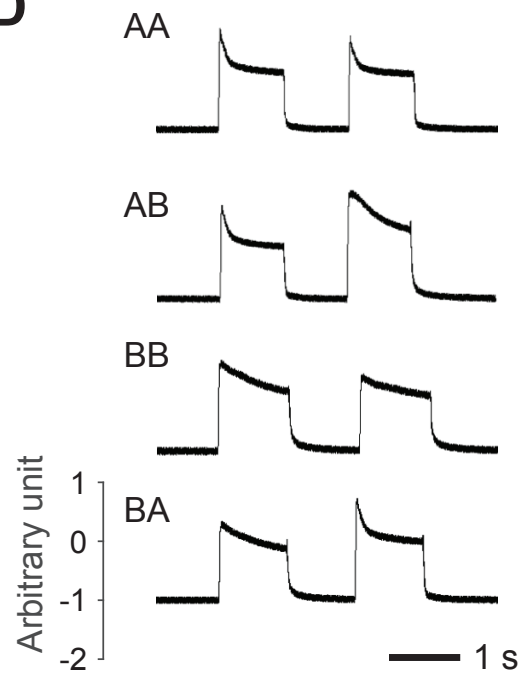

$\mathbf{F}$
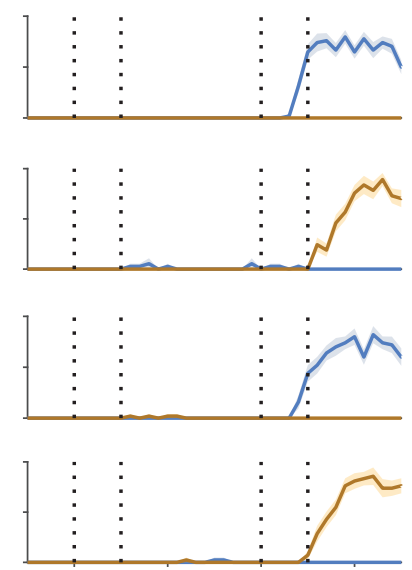

G Premature lick rate (licks/s)

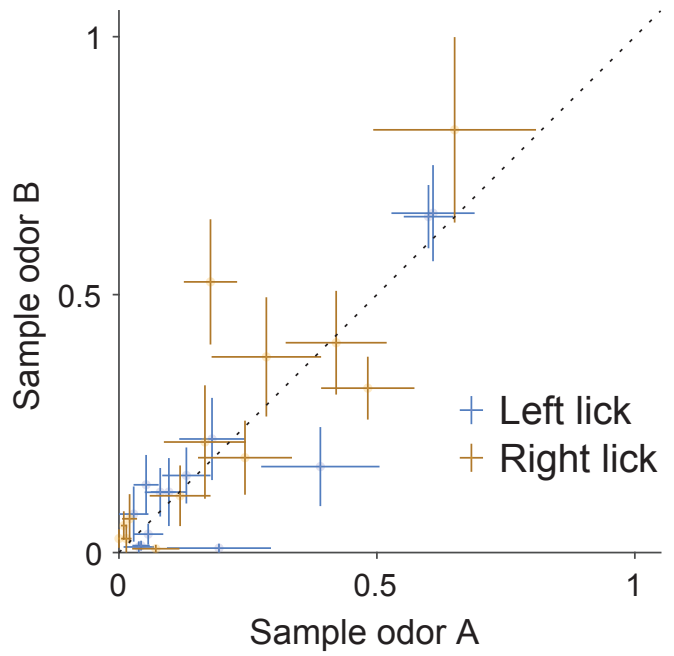




\section{$712 \quad$ Figure S1: Mouse training on the DMS task. Related to Figure 1.}

713 (A) Cumulative proportion of animals that reach training criterion as a function of training days (n

$714=41$ ). Training days only include the days in which mice were presented with all four trial types.

715 (B) Progression of each mouse's performance on the DMS task. Each line represents the data from

716 one animal; the green dot indicates the day on which they reached criterion performance.

717 (C) Performance of animals on a set of novel odors, $C / D \times C / D$. The animals $(n=5)$ were

718 previously trained to criterion on the $\mathrm{A} / \mathrm{B} \times \mathrm{A} / \mathrm{B}$ task. The poor performance is characterized by a

719 smaller proportion of correct choices and a large proportion of "no choice" trials.

720 (D) Time course of the sample and test odor delivery as measured by a photo-ionization detector.

721 The measurements were taken in the training phase when sample and test epochs were $1 \mathrm{~s}$.

722 (E, F) Premature licks are indistinguishable for the two sample odors. Lick rates of two example

723 animals, grouped by trial type. Only correct trials are shown. The lines and shadings indicate mean

724 and s.e.m. $(\mathrm{n}=47-79$ trials $)$.

725 (G) Mean lick rates during the sample and delay epochs for trials in which the sample odor was A,

726 plotted against the rates for sample odor B. Each point represents data from one animal $(n=13)$.

727 Error bars are s.e.m.

728

729 
bioRxiv preprint doi: https://doi.org/10.1101/757104; this version posted September 3, 2019. The copyright holder for this preprint (which was not certified by peer review) is the author/funder, who has granted bioRxiv a license to display the preprint in perpetuity. It is made available Figure S2
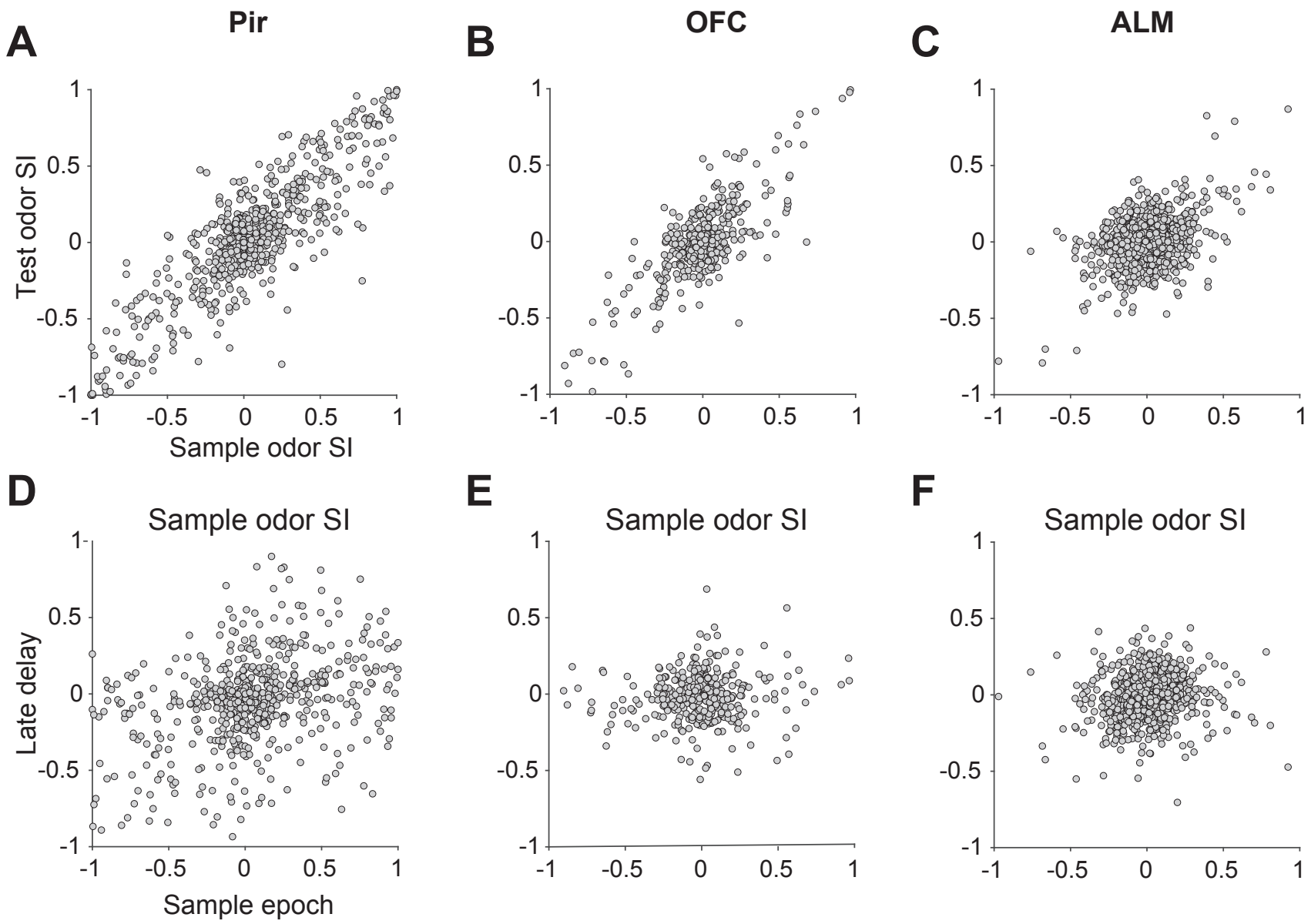

E
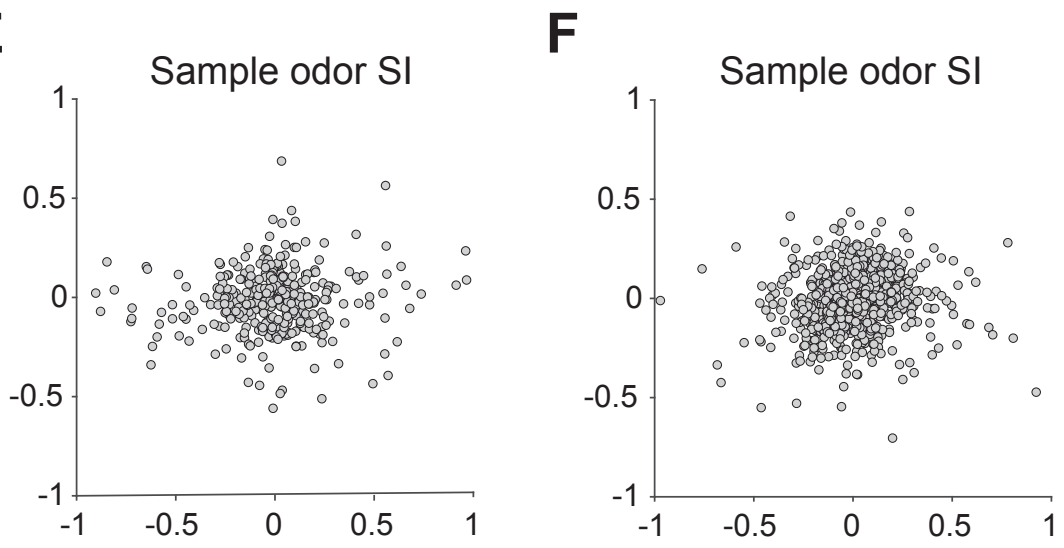

G
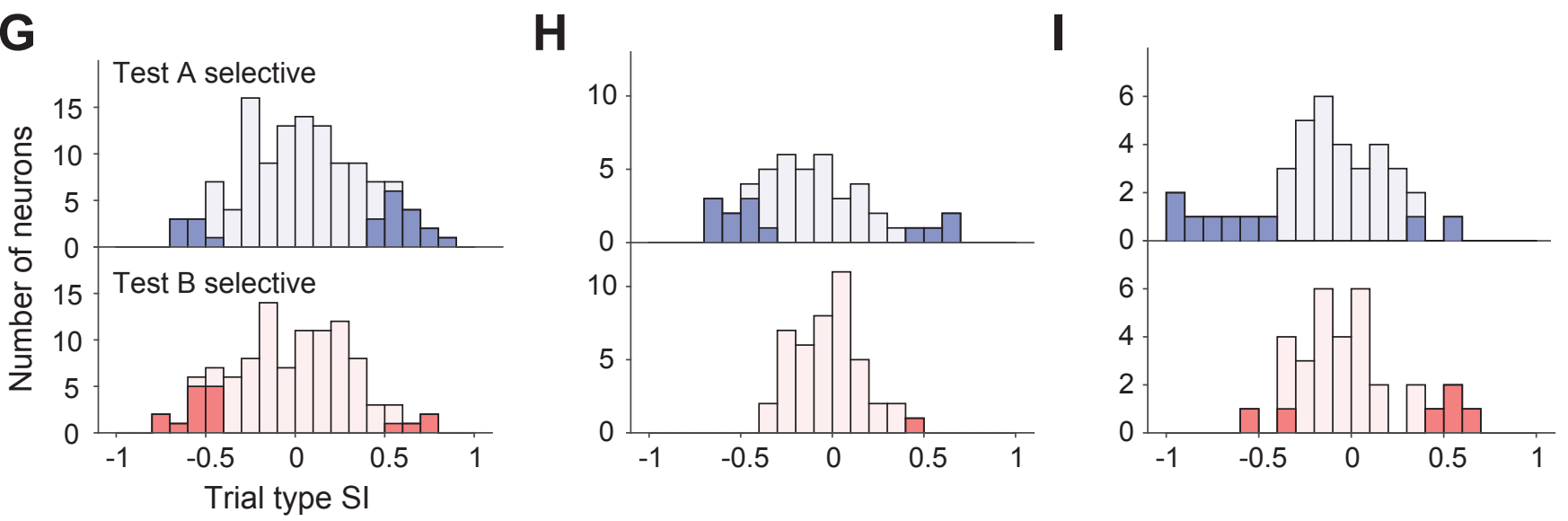

J

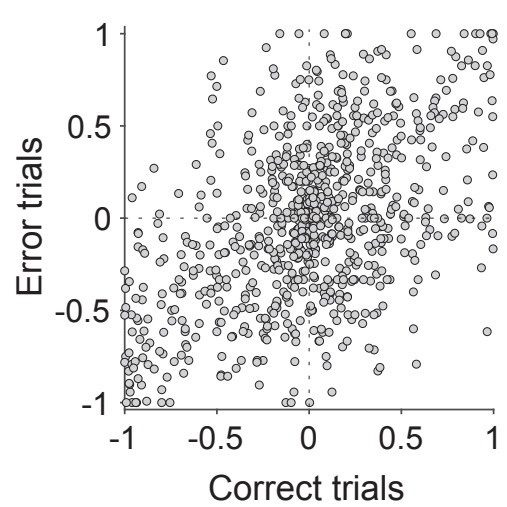

K

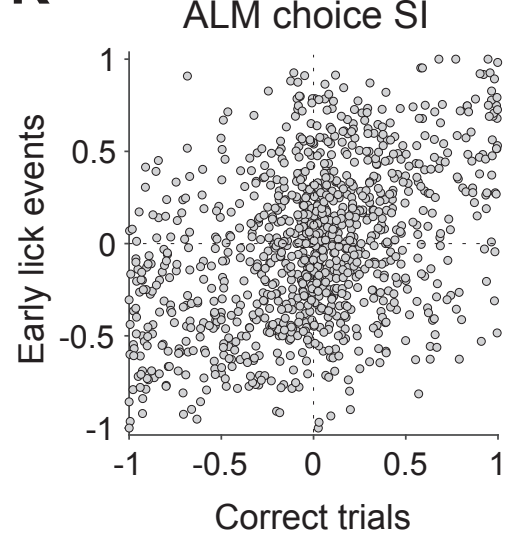

L

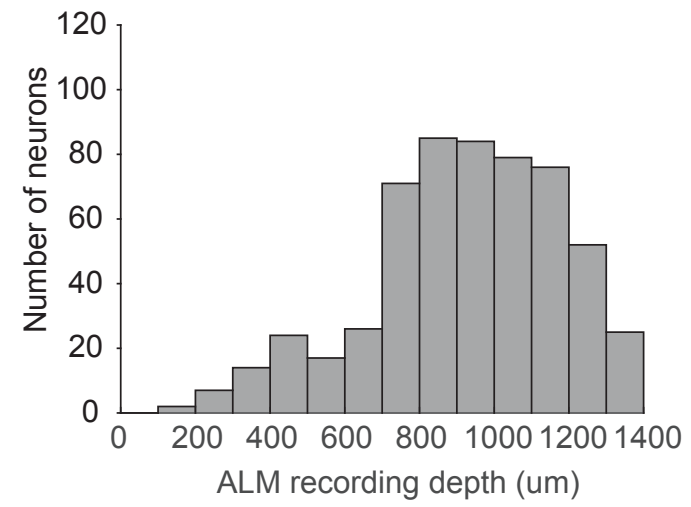




\section{Figure S2: Selectivity of task-related variables in Pir, OFC and ALM and the depth of ALM}

\section{1 neurons. Related to Figure 2.}

732 The three brain areas correspond to the left, middle and right columns of panels (A-I).

733 (A-C) Selectivity indices of Pir, OFC, and ALM neurons for sample and test odors (see Methods).

734 Pearson's correlation $R$ values: Pir, $0.86(P=1.6 \mathrm{e}-184)$; OFC, $0.79(P=1.3 \mathrm{e}-82)$; ALM, $0.41(P$

$735=5.9 \mathrm{e}-45)$

736 (D-F) Selectivity indices of neurons for sample odor in the sample epoch and in the late delay.

737 Pearson's correlation $R$ values: Pir, $0.34(P=9.5 \mathrm{e}-19)$; OFC, $0.076(P=0.14)$; ALM, $0.12(P=$ $738 \quad 5.7 \mathrm{e}-5)$

739 (G-I) Selectivity indices of neurons for trial type (see Methods). Only test odor selective neurons

740 are included. Top: selectivity indices of test odor A-preferring neurons for sample odor identity; a

741 positive value indicates a preference for AA trials and, a negative value indicates a preference for

742 BA trials. Shading denotes statistical significance (Mann-Whitney U test, $P<0.01$, two-tailed).

743 Bottom: same for test odor B-preferring neurons.

744 (J) Choice selectivity indices of ALM neurons in correct and error trials. Pearson's correlation, $R$ $745=0.51, P=4.6 \mathrm{e}-53$.

746 (K) Choice selectivity indices of ALM neurons in correct trials and in early lick events. $R=0.42$,

$747 \quad P=4.6 \mathrm{e}-47$.

748 (L) The depth of the recorded ALM units measured from the pial surface. 
bioRxiv preprint dol: https://doi.org/10.1101/757104; this version posted September 3, 2019. The copyright holder for this preprint (which was not certified by peer review) is the author/funder, who has granted bioRxiv a license to display the preprint in perpetuity. It is made available Figure S3 under aCC-BY-NC-ND 4.0 International license.

A

$0 \mathrm{~mm} \quad 0.5 \mathrm{~mm} 1.0 \mathrm{~mm} 1.5 \mathrm{~mm} 2.0 \mathrm{~mm}$

$10.0 \mathrm{~mW}$

$5.0 \mathrm{~mW}$

$1.5 \mathrm{~mW}$

$0.5 \mathrm{~mW}$

$0 \mathrm{~mW}$
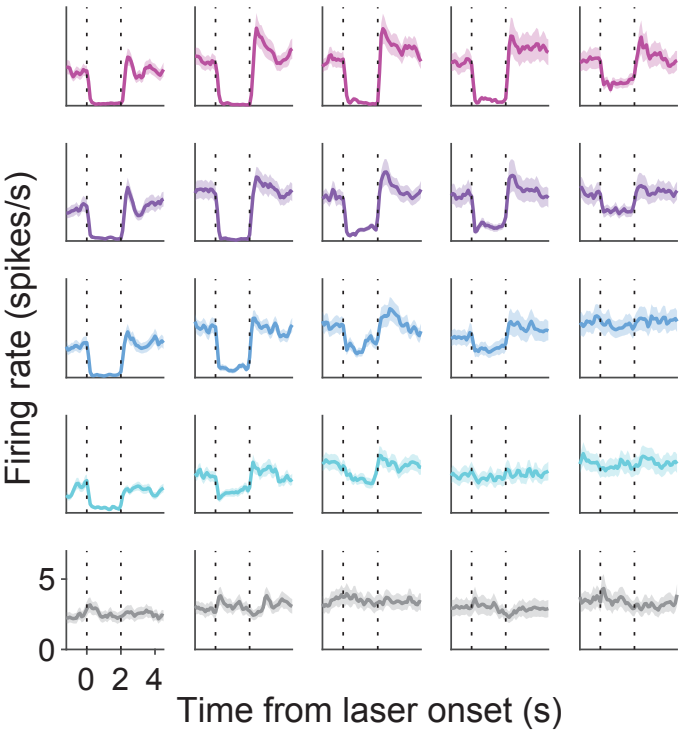

C

$10.0 \mathrm{~mW}$

$5.0 \mathrm{~mW}$

$1.5 \mathrm{~mW}$

$0.5 \mathrm{~mW}$

$0 \mathrm{~mW}$
B

$0 \mathrm{~mm} \quad 0.5 \mathrm{~mm} 1.0 \mathrm{~mm} 1.5 \mathrm{~mm} 2.0 \mathrm{~mm}$
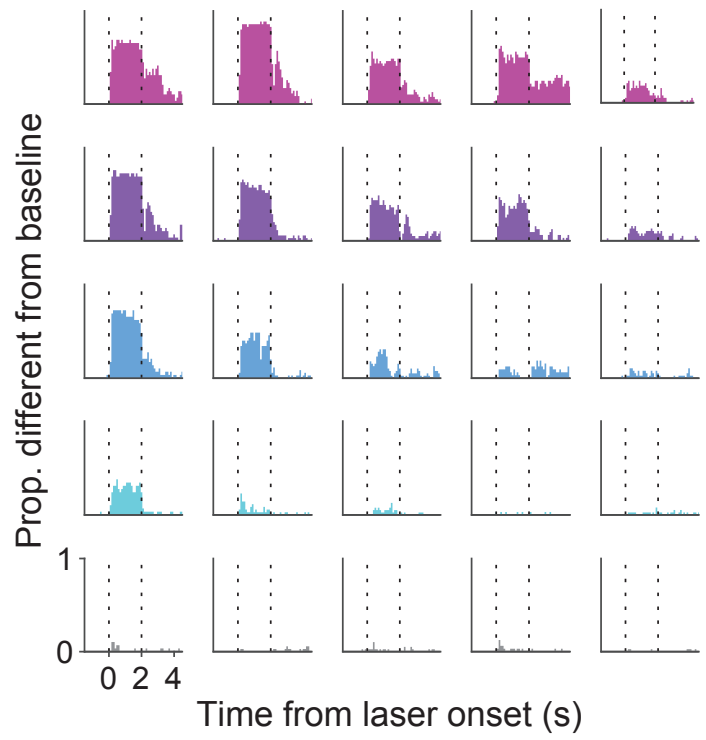


\section{$751 \quad$ Figure S3: Calibration of laser power for photoinhibition. Related to Figure 4.}

752 (A) Mean firing rate of ALM neurons over a 2 s period of photoinhibition $(n=74)$. Shading

753 indicates s.e.m. The rows denote different laser powers and the columns indicate the distance of

754 the recording sites from the center of the laser spot along the cortical surface. The $2 \mathrm{~s}$ illumination

755 includes a power ramp-down in the final $250 \mathrm{~ms}$.

756 (B) Proportion of neurons with firing rates that are significantly different from baseline (Mann-

757 Whitney U test, $P<0.01)$.

758 (C) Normalized mean firing rate of neurons during photoinhibition, plotted as a function of cortical

759 depth from the pial surface. The column labels are the same as in (A). Each point represents data

760 from one neuron. The lines are linear fits. The firing rates were normalized to the average response

761 in a 1s baseline period before laser onset. Only neurons with baseline firing rate $>1$ spikes/s were

762 included. 
bioRxiv preprint doi: https://doi.org/10.1101/757104; this version posted September 3, 2019. The copyright holder for this preprint (which was not certified by peer review) is the author/funder, who has granted bioRxiv a license to display the preprint in perpetuity. It is made available

Figure S4

A

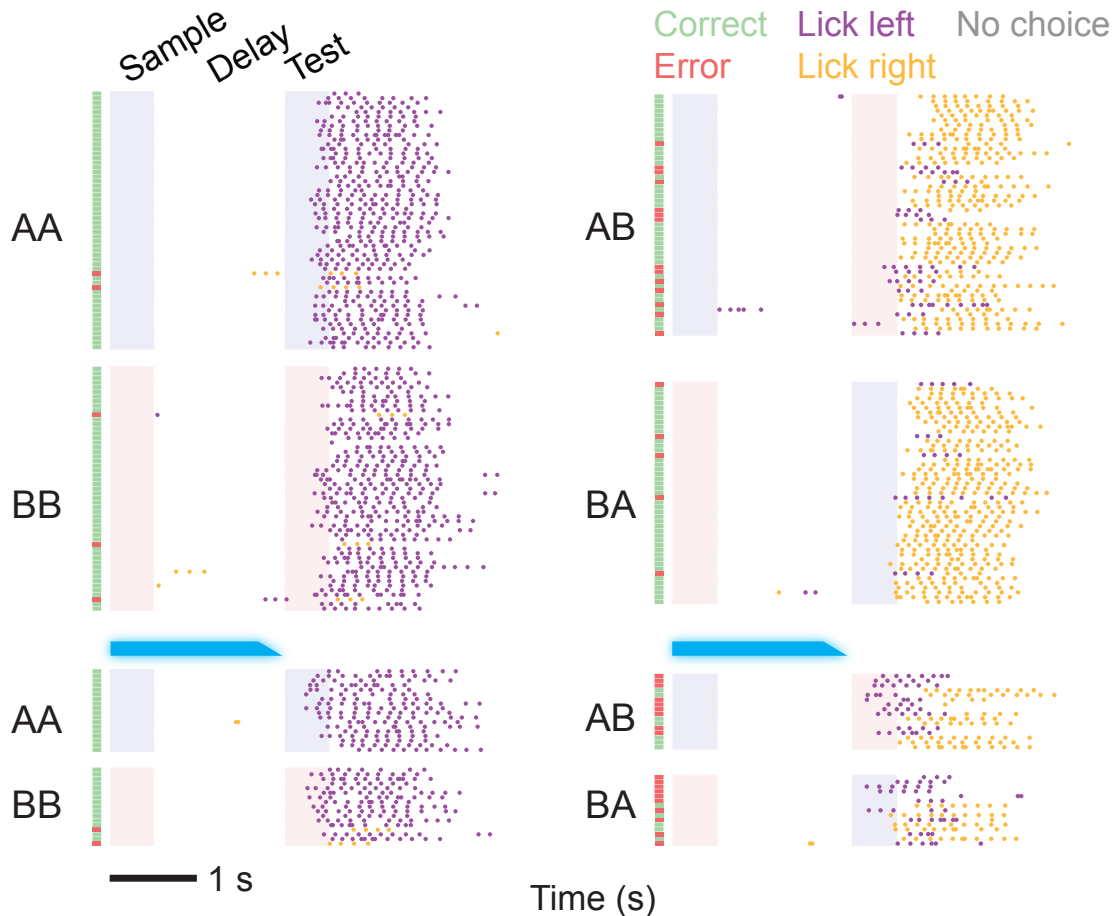

B

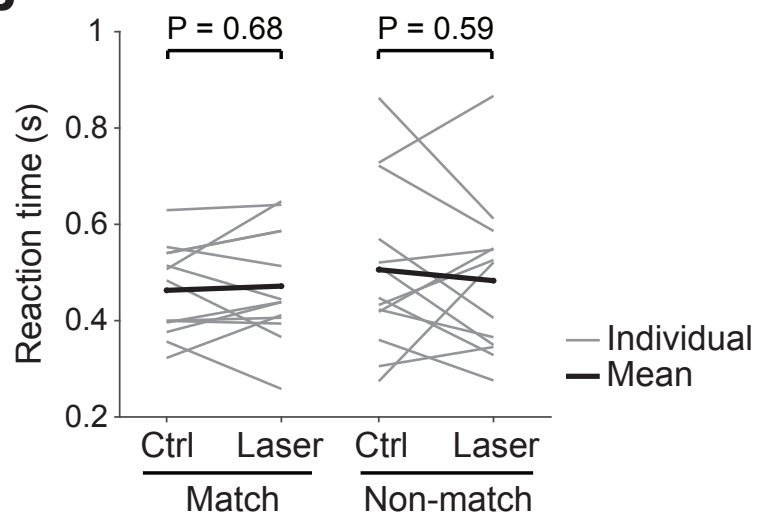

C

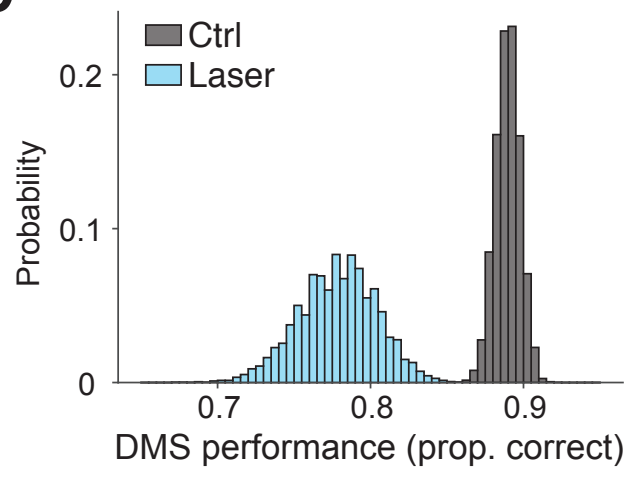




\section{Figure S4: Licking and side bias in ALM inactivation. Related to Figure 4.}

766 (A) An example behavioral session with and without ALM inactivation. The animal still licked to

767 the two ports but made more errors. The top two rows are control trials and the bottom two rows

768 are inactivation trials. All the trials were randomly presented but grouped here for plotting. The

769 blue bars indicate the timing of the photoinhibition.

770 (B) Reaction time in match and non-match trials with and without ALM inactivation. Each thin

771 line represents data from one animal; the thick lines connect the means from 13 animals. Control

772 and laser groups were compared with paired $t$-test.

773 (C) Estimation of behavioral impairment not accounted for by a side bias. Monte Carlo methods

774 were used to estimate the change in accuracy that was not attributable to a bias. We simulated

775 datasets matched in size to the experiments in Fig. 4B, using the logistic fits to the data but with

776 the laser induced bias set to zero ( $\beta_{3}=0$, Eqs. 7 and 8 , see Methods). The histograms show

777 distributions of Proportion Correct for control and laser from 10,000 simulations. The difference

778 in expectations, $0.89 \pm 0.008$ (mean \pm standard deviation) to $0.78 \pm 0.025$ correct $(P=1.8 \mathrm{e}-5, t$ -

779 test), is a conservative estimate of the impairment that is not induced by a laser induced bias. The

780 estimate is conservative because bias could change as a consequence of uncertainty associated

781 with impairment on the task. The simulation complements the logistic regression analysis in which

782 choices are coded to maximize the effect of laser induced inactivation on bias (Eqs. 7 and 8).

783 Estimated coefficients are $\beta_{1}=2.10 \pm 0.059, P=4.0 \mathrm{e}-281 ; \beta_{2}=1.00 \pm 0.11, P=7.3 \mathrm{e}-19 ; \beta_{3}=-$

$7840.84 \pm 0.11, P=1.5 \mathrm{e}-13$. Note that sensitivity on the task is significantly impaired by inactivation $785\left(\beta_{3}<0\right)$. 
Figure S5 under aCC-BY-NC-ND 4.0 International license.

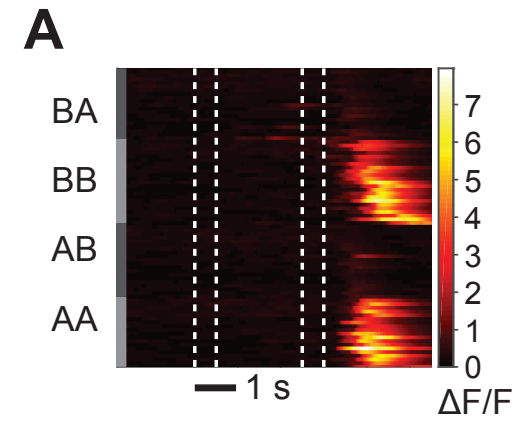

D

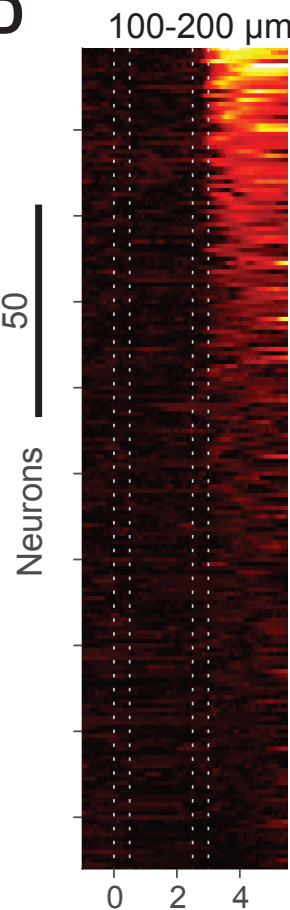

Time from sample onset (s)
B

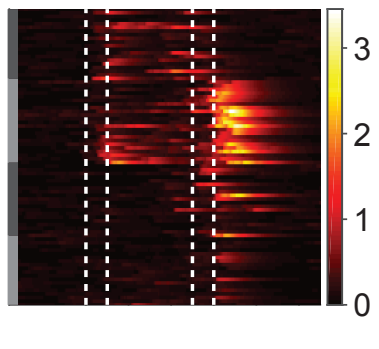

C
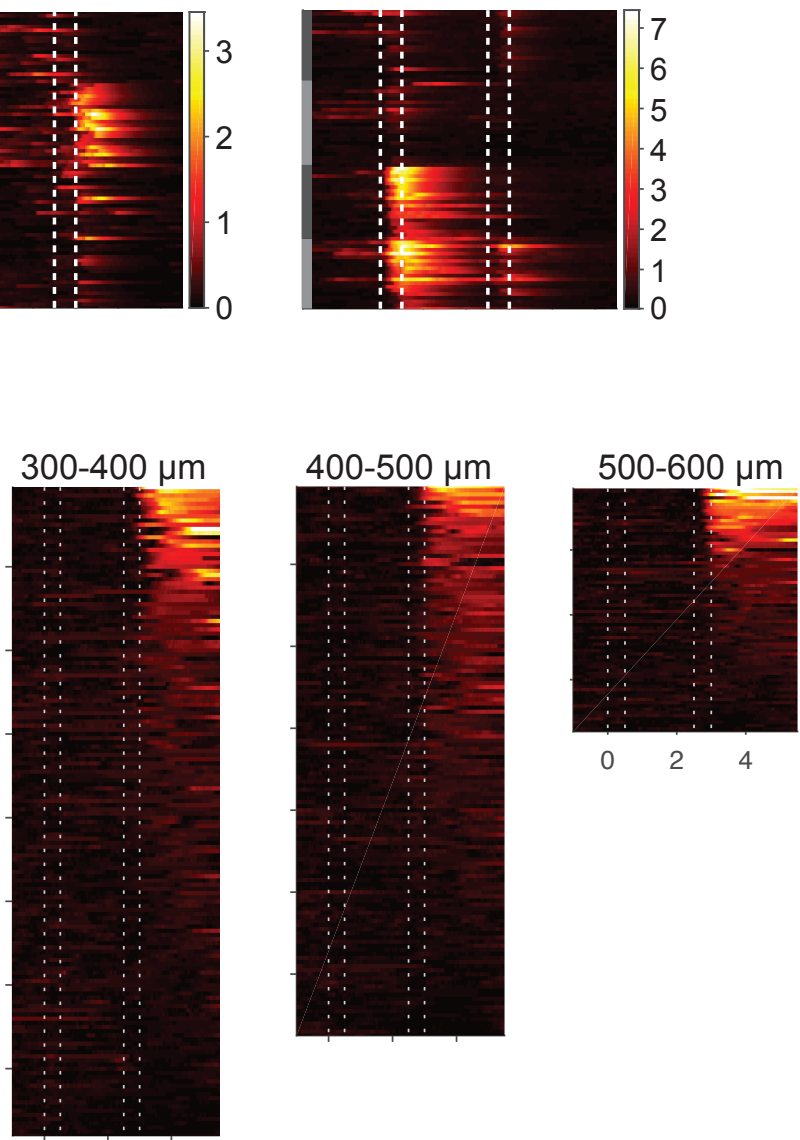

$\begin{array}{lll}0 & 2 & 4\end{array}$

\section{Standardized selectivity}

$0 \quad 1 \quad 2 \quad 3$ 
788 Figure S5: Calcium responses and selectivity of ALM neurons. Related to Figure 5.

789 (A-C) $\Delta \mathrm{F} / \mathrm{F}$ of three example ALM neurons. Each row is a trial, grouped by trial type.

790 (D) Standardized choice selectivity of ALM neurons across different cortical depths (see Methods).

791 Each row is a neuron, sorted by the mean standardized choice selectivity within $2.5 \mathrm{~s}$ of test onset.

792

793 
bioRxiv preprint doi: https://doi.org/10.1101/757104; this version posted September 3, 2019. The copyright holder for this preprint (which was not certified by peer review) is the author/funder, who has granted bioRxiv a license to display the preprint in perpetuity. It is made available Figure S6 under aCC-BY-NC-ND 4.0 International license.

A

Neuron $1,2,3 \ldots \rightarrow$

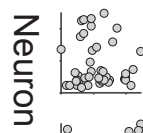

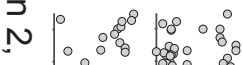

$\omega$

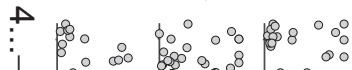

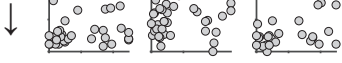

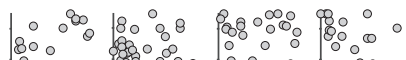

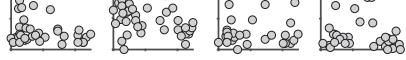

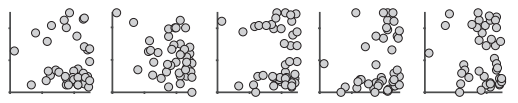

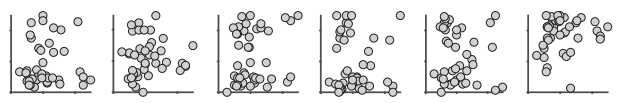

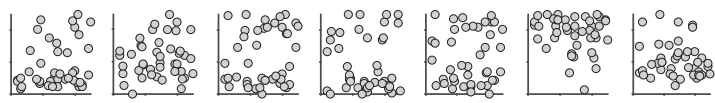

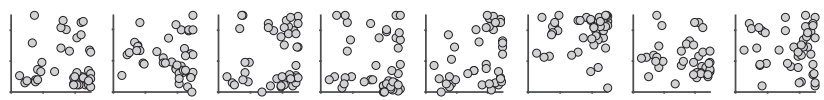

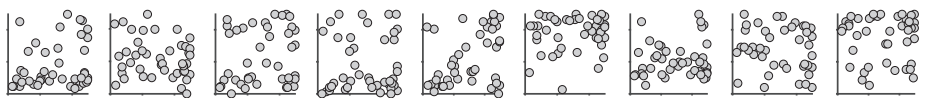

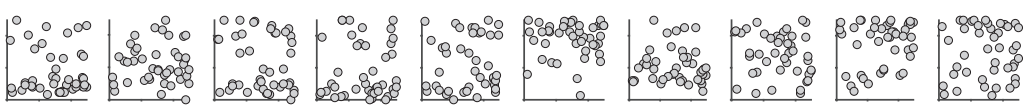

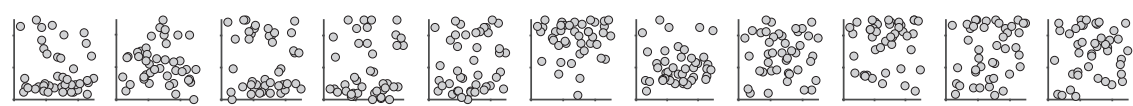

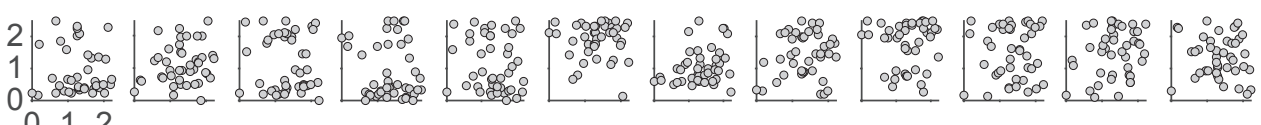

Time of peak response (s)

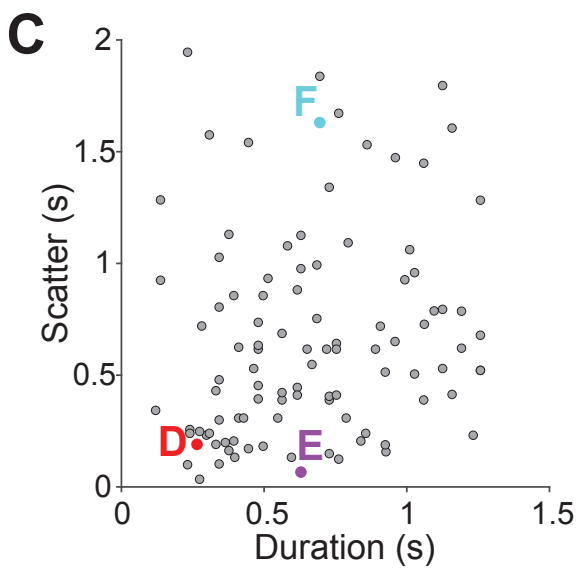

Time from sample onset (s)
E $\quad F$
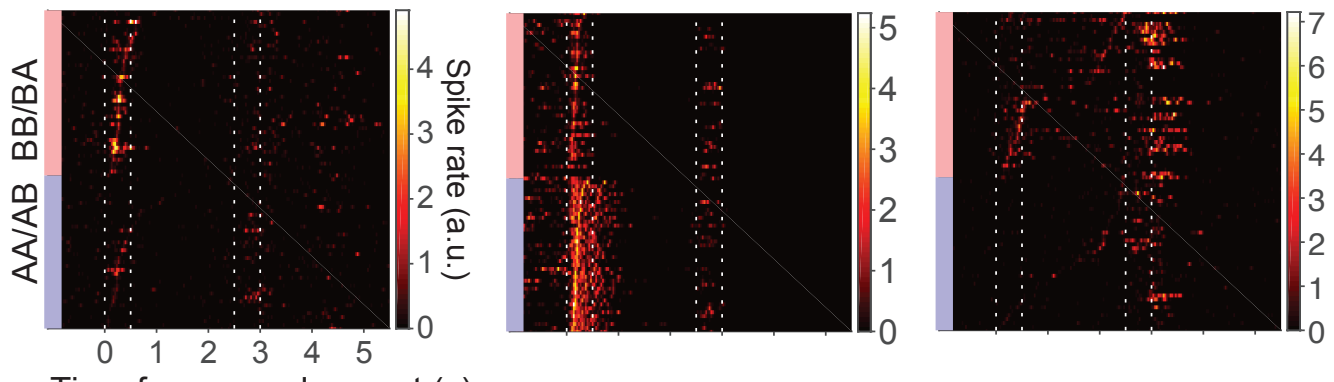


\section{Figure S6: Correlation of peak response time and deconvolved spike rates. Related to Figure}

7955.

796 (A) Correlation of the peak response time between simultaneously recorded neuron pairs from a

797 session. Only sample odor B selective neurons with a peak time scatter (see Fig. 6E) over $0.6 \mathrm{~s}$ are

798 included.

799 (B) Distribution of the correlation coefficients of the peak response from all neuron pairs. The

800 shading indicates coefficients that are significantly different from chance $(P<0.01$; Fisher $\mathrm{z}$,

801 uncorrected for multiple comparisons). The red dashed curve shows the distribution of correlation

802 coefficients under the null hypothesis (estimated from a shuffle control; see Methods).

803 (C) The duration and scatter of L2 sample-selective neurons using deconvolved spike rates. The

804 three colored points correspond to the neurons shown in panels (D-F).

805 (D-F) The deconvolved spike rates of three example neurons shown in Fig. 6B-D, respectively.

806 Each row is a trial, grouped by sample odor identity and sorted by peak response time to sample 807 odor. 


\section{Key Resources Table}

\begin{tabular}{|c|c|c|}
\hline REAGENT OR RESOURCE & SOURCE & IDENTIFIER \\
\hline \multicolumn{3}{|l|}{$\begin{array}{l}\text { Experimental models: } \\
\text { Organisms/Strains }\end{array}$} \\
\hline Mouse: wild type C57BL/6J & $\begin{array}{l}\text { Jackson } \\
\text { Laboratory }\end{array}$ & 000664 \\
\hline Mouse: VGAT-ChR2-EYFP & $\begin{array}{l}\text { Jackson } \\
\text { Laboratory }\end{array}$ & 014548 \\
\hline $\begin{array}{l}\text { Mouse: Ai93(TITL- } \\
\text { GCaMP6f)-D;CaMK2a-tTA }\end{array}$ & $\begin{array}{l}\text { Jackson } \\
\text { Laboratory }\end{array}$ & 024108 \\
\hline Mouse: Emx1-IRES-cre+/+ & $\begin{array}{l}\text { Jackson } \\
\text { Laboratory }\end{array}$ & 005628 \\
\hline \multicolumn{3}{|l|}{ Electrophysiology } \\
\hline $\begin{array}{l}32 \text { channel, acute Buzsaki32 } \\
\text { probe }\end{array}$ & Neuronexus & Buzsaki32-A32 \\
\hline $\begin{array}{l}32 \text { channel, chronic Buzsaki32 } \\
\text { probe }\end{array}$ & Neuronexus & Buzsaki32-H32_21mm \\
\hline 32 channel, acute Poly3 probe & Neuronexus & A1x32-Poly3-5mm-25s-177-A32 \\
\hline $\begin{array}{l}64 \text { channel, acute } 1 \times 64 \mathrm{H} 3 \\
\text { probe }\end{array}$ & Tim Harris & $\begin{array}{l}\text { https://www.cambridgeneurotech.com/silicon- } \\
\text { probes }\end{array}$ \\
\hline \multicolumn{3}{|l|}{ Software } \\
\hline MATLAB & Mathworks & https://www.mathworks.com \\
\hline LabVIEW & $\begin{array}{l}\text { National } \\
\text { Instrument }\end{array}$ & $\begin{array}{l}\text { https://www.ni.com/en-us/shop/labview/labview- } \\
\text { details.html }\end{array}$ \\
\hline SIMA & Kaifosh et al. & https://www.losonczylab.org/software \\
\hline CNMF & $\begin{array}{l}\text { Pnevmatikakis et } \\
\text { al. }\end{array}$ & $\begin{array}{l}\text { https://www.cell.com/neuron/fulltext/S0896- } \\
\text { 6273(15)01084-3 }\end{array}$ \\
\hline Python & Python & https://www.python.org \\
\hline Tensorflow & Tensorflow & https://www.tensorflow.org \\
\hline
\end{tabular}




\section{Animals}

814 Animals were maintained on 12h: 12h light/dark cycle with food and water available ad libitum.

815 Animal care and experiments were carried out in accordance with the NIH guidelines and approved

816 by the Columbia University Institutional Animal Care and Use Committee (IACUC).

818 Mice were water-restricted during the training and testing phases. Experimental sessions were 1-

8192 hours, during which mice received $0.5-1.5 \mathrm{ml}$ of water. Animals received supplemental water as

820 necessary to maintain their body weights. Aseptic surgeries were carried out under ketamine (100

$821 \mathrm{mg} / \mathrm{kg}) / \mathrm{xylazine}(10 \mathrm{mg} / \mathrm{kg})$ or $1-3 \%$ isoflurane anesthesia. Buprenorphine $(0.05-0.1 \mathrm{mg} / \mathrm{kg})$ and

822 carprofen $(5 \mathrm{mg} / \mathrm{kg})$ were administered for postoperative analgesia.

823

824 This study is based on data from 41 mice (both males and females, 2-8 months old). 5 C57BL/6J

825 and 6 VGAT-ChR2-EYFP (Jackson Laboratory, JAX 014548) mice were used for

826 electrophysiology recording. 2 untrained VGAT-ChR2-EYFP mice were used to characterize the

827 inhibition at different laser powers. 29 VGAT-ChR2-EYFP mice were used for inhibition

828 experiments, 6 of which were also used for simultaneous recording during inhibition. Two-photon

829 imaging data were collected from 5 Emx1-cre+/-; TITL-GCaMP6f+/-; CaMK2a-tTA+ mice.

830 These mice were created by crossing Ai93(TITL-GCaMP6f)-D;CaMK2a-tTA (JAX 024108) to

831 Emx1-IRES-cre+/+ (JAX 005628) line.

\section{Behavior training}

834 Before training, mice were implanted with a custom-made titanium head post (Guo et al., 2014b).

835 The scalp and periosteum over the dorsal surface of the skull were removed, and a head post was 
836 placed on the skull, aligned with the lambda suture and cemented in place with C\&B metabond

837 (Parkwell, S380). After at least 2-3 days of recovery, animals were water-restricted and

838 accustomed to head-fixation following procedures described in Guo et al., 2014 (Guo et al., 2014b)

839 and then trained on a custom-assembled apparatus.

841 Odorants were delivered with a custom-made olfactometer and custom-written LabVIEW

842 programs (National Instruments). (+)- $\alpha$-Pinene (odor A, Sigma-Aldrich, 268070), cis-3-Hexen-1-

843 ol (odor B, Sigma-Aldrich, W256307), (R)-(+)-Limonene (odor C, Sigma-Aldrich, 183164), and

844 Methyl butyrate (odor D, Sigma-Aldrich, 246093) were chosen for their lack of innate valence to

845 mice (Root et al., 2014) and their low adhesion to the surface of the olfactometer. The odorants

846 were diluted 100-fold in mineral oil (Fisher Scientific, O121-1) and then loaded on syringe filters

847 (GE healthcare, 6888-2527 or 6823-1327). The air flow was maintained at $1.0 \mathrm{~L} / \mathrm{min}$. We

848 confirmed the rapid kinetics (Fig. S1D) of these odorants with photo-ionization detector (Aurora

849 Scientific, 200B). Over the course of a behavioral session, the odorants on the syringe filters

850 gradually deplete. Thus animals were unlikely to rely on the absolute concentration of the odorants

851 which changed constantly, but rather the identity of the odors, as evidenced by the stable sensory

852 responses in Pir over a session (Fig. 2A).

854 During training, mice were presented with a sample odor (1.0 s duration) and a test odor (1.0 s)

855 separated by a delay epoch $(1.0 \mathrm{~s})$. After hearing an auditory "go" cue $(0.1 \mathrm{~s}, 5 \mathrm{KHz}$ pure tone),

856 they were free to report their decision by licking to one of the two syringe ports positioned in front

857 of their mouth, and collect a water reward at the same port if they were correct. Many animals

858 started licking in the test epoch, which was permitted, but only the licks during the $2 \mathrm{~s}$ "response 
859 window" following the "go" cue were considered their choice. To prevent mice from "probing"

860 for the correct port by rapidly switching between the two ports, we required mice to commit to

861 their choices. A choice is scored as correct only when the first two licks were on the correct port.

862 If they first licked on the incorrect port, that trial was scored as an error. If they licked once on the

863 correct port and then on the incorrect port, that trial was also scored as an "error". If they did not

864 lick during the "response window" or only licked once on the correct port, that trial was scored as

865 "no choice".

867 The two odorants give rise to four unique pairs of sample and test odors (AA, $\mathrm{AB}, \mathrm{BB}$, and $\mathrm{BA}$ ),

868 or trial types, and were randomly presented in each session. The match trials (AA, BB) were

869 rewarded on the left port and non-match trials $(\mathrm{AB}, \mathrm{BA})$ on the right. Mice were punished by a

870 brief timeout (3-8 s) when they made an error. "No choice" trials were rare and typically occurred

871 in the very early training stage or at the end of a session when the mice were sated. Animals

872 completed this training stage when they achieved a criterion of at least $80 \%$ correct for each trial

873 type in a single session. They then underwent additional training to suppress "premature" licking

874 before the test epoch. Such early licks were punished by an immediate 0.1-0.2 s siren (RadioShack,

875 273-079), followed by a 0.5-1.0 s pause in that trial, and a longer inter-trial interval. We required

876 the proportion of trials with premature licking to be less than $\sim 20 \%$. After achieving these

877 milestones (median 25 days; IQR 21-31 days) the sample and test durations were reduced from

$8781.0 \mathrm{~s}$ to $0.5 \mathrm{~s}$, and the delay was increased from $1.0 \mathrm{~s}$ to $1.5-4.0 \mathrm{~s}$ depending on the experiment (1.5

$879 \mathrm{~s}$ in extracellular recording, $2.0 \mathrm{~s}$ in imaging experiments, $1.5,2.5$ or $4.0 \mathrm{~s}$ in optogenetic

880 inactivation). Mice performed at 90\% correct (interquartile range 88-92\%) when they entered the

881 testing phase. 


\section{Electrophysiology}

884 Extracellular recordings were made acutely or chronically in head-fixed animals using 32- or 64-

885 channel silicon probes (Buzsaki32 and Poly3, NeuroNexus; 1×64 acute H3 probe, HHMI). The

886 probes were targeted stereotaxically to Pir, OFC and ALM using Bregma coordinates as follows:

887 Pir: AP 1.9-2.4 mm, ML 1.5-2.1 mm, DV 2.3-2.9 mm; OFC: AP 2.2-2.8 mm, ML 0.7-1.2 mm, DV

890 In acute recordings, a small craniotomy $(0.3-0.8 \mathrm{~mm}$ in diameter $)$ was made over the targeted area

891 before the recording session. Recording depth from the pial surface was inferred from

892 micromanipulator reading. After each recording session, the brain surface was covered with

893 silicone gel (3-4680, Dow Corning) and Kwik-Sil (World Precision Instruments). In chronic

894 recordings, the probe (Buzsaki32-H32_21mm, NeuroNexus) was attached to a custom-made

895 microdrive which allows for advancement of the shanks. The microdrive was then implanted and

896 cemented with C\&B metabond and dental acrylic (Lang Dental Jet Repair Acrylic, 1223CLR). We

897 advanced the shanks by $50 \mu \mathrm{m}$ per day at the end of each recording session.

\section{Two-photon calcium imaging}

900 Calcium imaging was performed on Emx1-cre+/-; TITL-GCaMP6f+/-; CaMK2a-tTA+ mice. A

901 square craniotomy ( $2 \mathrm{~mm}$ side) was made above left or right ALM, along the superior sagittal sinus

902 and the inferior cerebral vein. The imaging window was constructed from three stacked layers of

903 custom-cut coverglass (CS-3S, Warner Instruments) and cemented with C\&B metabond. Animals

904 were allowed 1-2 weeks of recovery before the imaging sessions began. Images were acquired 
with a Bruker Ultima two-photon microscope under resonant galvo scanning mode. The light source was a femtosecond pulsed laser (Chameleon Vision II, Coherent). The objective was a 16X water immersion lens (Nikon, 0.8 NA, 3mm working distance). GCaMP6f was excited at 920nm and images (512 x 512 pixels, $\sim 820 \mu \mathrm{m}$ x $820 \mu \mathrm{m}$ field of view) were acquired at $\sim 30 \mathrm{~Hz}$.

\section{Photostimulation}

911 Animals were prepared with a clear skullcap to achieve optical access to ALM (Guo et al., 2014a).

912 Briefly, after removing the scalp and periosteum over the dorsal surface of the skull, a layer of 913 cyanoacrylate adhesive (Krazy glue, Elmer's Products Inc.) was directly applied to the intact skull.

914 The entire skull was then covered with a thin layer of the clear dental acrylic (Lang Dental) with 915 a head post cemented over the lambda suture. Before photostimulation sessions, the dental acrylic 916 was polished (0321B, Shofu Dental Corporation) and covered with a thin layer of clear nail polish 917 to reduce glare (Part No. 72180, Electron Microscopy Sciences). Light from a 473nm laser (MLL918 FN-473-50mW, Ultralasers, Inc.) was directed to an optic fiber and split into two paths (FCMH2919 FCL, Thorlabs, $\varnothing 200 \mu \mathrm{m}$ Core, 0.39 NA). The two optic fibers were positioned over ALM on each 920 hemisphere. The light transmission through the skullcap is $\sim 50 \%$ in average power, as measured 921 directly with a light meter (PM100D, Thorlabs) with freshly dissected skullcap, consistent with 922 previous measurements (Guo et al., 2014a).

924 We used $40 \mathrm{~Hz}$ photostimulation with a sinusoidal temporal profile ( $3 \mathrm{~mW}$ average power as light 925 reaches the skull, $\sim 1.5 \mathrm{~mW}$ on the brain surface). The photoinhibition inactivated a cortical area 926 of $\sim 1 \mathrm{~mm}$ radius, as the population firing rate drops to $\sim 50 \%$ at $1 \mathrm{~mm}$ away from the center of the 927 laser beam (Fig. S3A). To reduce rebound excitation after laser offset, we included a 250 ms linear 
928 power ramp-down at the end of the photostimulation (Fig. 4A) unless otherwise indicated. In the

929 interleaved $\mathrm{A} / \mathrm{B} \times \mathrm{A} / \mathrm{B} \& \mathrm{C} \times \mathrm{C} / \mathrm{D}$ experiment, the delay epoch was extended to $4 \mathrm{~s}$ while sample

930 and test epochs were kept at $0.5 \mathrm{~s}$ each. Here we used a $500 \mathrm{~ms}$ ramp-down at the end of the $4 \mathrm{~s}$

931 photostimulation, which terminates $500 \mathrm{~ms}$ before the test odor onset to allow more recovery time.

932 To prevent the mice from distinguishing photostimulation trials from control trials, a masking flash

933 (40 Hz sinusoidal profile) was delivered with $470 \mathrm{~nm}$ LED (Luxeon Star) and LED driver (SLA-

934 1200-2, Mightex Systems) in front of the animals' eyes on all trials. The masking flash was not

935 phased locked to the photostimulation, began at sample onset and lasted until the end of test,

936 covering the entire stimulus and delay epochs in which photostimulation could occur.

938 For the experiments in which we varied the duration of inactivation (Fig. 4E), photostimulation

939 was either limited to $(i)$ the $0.5 \mathrm{~s}$ sample epoch and the first $1.5 \mathrm{~s}$ of the $2.5 \mathrm{~s}$ delay epoch, or $(i i)$ the

940 last 1.0 s of the delay epoch, or (iii) the entire sample plus delay epoch. These inactivation trials

941 were randomly interleaved to constitute $25 \%$ of all trials. For each animal, multiple (2-3)

942 behavioral sessions were pooled to collect at least 10 trials for each of the four trial types and the

943 three inactivation durations.

945 Simultaneous photostimulation and recording

946 We calibrated the laser power for ALM and OFC inactivation by recording from these two areas

947 during photoinhibition in awake animals. For ALM inactivation, we positioned an acute 1x64 H3

948 probe at various distances from the optic fiber over ALM (0-2.0 mm in $0.5 \mathrm{~mm}$ increments). A

949 range of laser powers $(0.5 \mathrm{~mW}, 1.5 \mathrm{~mW}, 5.0 \mathrm{~mW}$, and $10.0 \mathrm{~mW})$ as well as controls were examined

950 at each location. We chose $1.5 \mathrm{~mW}$ power on the brain surface as it inactivates a cortical area of 
$951 \sim 1 \mathrm{~mm}$ radius and produces minimal rebound activity after laser offset (Fig. S3). For OFC

952 inactivation, an optic fiber was targeted to Bregma AP $2.5 \mathrm{~mm}$, ML $1.0 \mathrm{~mm}$, DV $1.4 \mathrm{~mm}$. The

953 recording probe was then positioned at AP $2.5 \mathrm{~mm}$, ML $1.5 \mathrm{~mm}$, DV 1.0-2.3 mm, where it is close

954 to the border of OFC with agranular insular cortex. We recorded the neural responses similarly at

955 a range of laser powers and chose $1.0 \mathrm{~mW}$ to silence OFC while minimizing the impact on

956 neighboring brain areas.

957

958 For simultaneous recording and inactivation when animals performed the DMS task, we used the

959 clear skullcap preparation for photoinhibition and made a small craniotomy lateral to the optic

960 fiber to insert the probe. The probe was advanced at $60-70^{\circ}$ angle from the horizontal plane at

961 Bregma AP 2.3-2.5 mm, ML 2.0-2.5 mm to record from ALM, OFC, and Pir at different depth.

963 Electrophysiology data analysis

964 The 32- or 64-channel recording data were digitized at $40 \mathrm{KHz}$ and acquired with OmniPlex D

965 system (Plexon Inc.) The voltage signals were high-pass filtered (200 Hz, Bessel) and sorted

966 automatically with Kilosort (Pachitariu et al., 2016). The clusters were then manually curated with

967 Phy GUI (Rossant et al., 2016) to merge spikes from the same units and to remove noises and units

968 that were not well isolated. Recording depths were inferred from micromanipulator readings in

969 acute recordings or microdrive turns in chronic recordings.

970

971 We determined sample odor selectivity for each neuron by comparing the spike counts during the

972 sample epoch (0.1-0.6 s from sample onset) or late delay (1.5-2.0 s from sample onset) between

973 sample odor A group (AA, AB) and sample odor B group (BA, BB). The $0.1 \mathrm{~s}$ time offset from 
974 the sample epoch accounts for the olfactometer valve time. A neuron is considered odor selective

975 if its responses to sample odor A and B are significantly different by a two-tailed Mann-Whitney

$976 \mathrm{U}$ test $(P<0.01$, not corrected for multiple comparisons). The selectivity index (SI) was computed

977 as follows for each unit: First, the trial-by-trial spike counts from the responses to odor A and B

978 were used to construct a Receiver Operating Characteristic (ROC). The area under the ROC

979 (AuROC) is the probability that a randomly sampled response associated with odor A is larger

980 than a randomly sampled response associated with odor B. The selectivity index is

$$
\mathrm{SI}=2\left(\operatorname{AuROC}-\frac{1}{2}\right)
$$

982 so that \pm 1 represents perfect discriminability (i.e., no overlap of the response counts) and 0

983 indicates chance-level separation. Positive SI connotes a preference for sample odor A. We

984 determined selectivity for test odor and choice in a similar fashion by comparing spike counts

985 during the test epoch (2.1-2.6 s from sample onset), with appropriate trial grouping. Positive SI for

986 choice connotes a preference for licking to the left spout.

988 The trial type selectivity in Fig. S2G-I was computed as follows. Neurons from each of the three

989 areas were first selected by their test odor selectivity. Only neurons that are test odor selective as

990 determined by a Mann-Whitney $U$ test $(P<0.01$, not corrected for multiple comparisons $)$ were

991 included in the subsequent analysis. These test odor selective neurons were then treated separately

992 based on their preference for test odor A or B. If a neuron prefers test odor A, its selectivity index

993 for trial type was computed based on its responses to AA vs. BA trials, in a similar fashion

994 described above. Positive SI connotes a preference for AA. If a neuron prefers test odor B, its trial

995 type selectivity is computed based on its response to $\mathrm{AB}$ vs. $\mathrm{BB}$, and positive SI connotes a 996 preference for $\mathrm{AB}$. 
Graphs depicting population odor selectivity of a brain area show the difference in firing rates associated with the preferred and nonpreferred odors (Fig. 3A, 4I-N). The preferred odor of each neuron was designated using a subset of the trials ( $n=5$ per odor), in the epoch under consideration

1001 (e.g., sample), based on the sign of the difference in the means, irrespective of statistical 1002 significance. These trials were then excluded and the odor selectivity was computed as the spike 1003 rate to preferred odor minus the nonpreferred odor on the remaining data in a sliding bin of 100 1004 ms. All neurons from each of the three areas are included in Fig. 3A (left graph). In Fig. 4I-N, all 1005 neurons from each of the three areas are included.

1007 For the decoding analysis (Fig. 3B-D, 4O-R and 6A, B), we trained a support vector machine 1008 (SVM) (Fan, Rong-En, 2008) with neural responses recorded simultaneously from Pir, OFC, or 1009 ALM to classify sample odor identity, trial type, or match/nonmatch. 48-120 neurons were 1010 simultaneously recorded from Pir, 16-82 neurons from OFC and 14-82 neurons from ALM. In Fig. 1011 3B, we used the spike counts in a sliding bin of $500 \mathrm{~ms}$ from 40 randomly selected neurons from 1012 each of the three areas at $100 \mathrm{~ms}$ steps. Sessions with insufficient number of simultaneously 1013 recorded neurons were excluded. In Fig. 3C, we used a $500 \mathrm{~ms}$ sliding bin with neural responses 1014 from all Pir neurons recorded in a session. In Fig. 3D, we used the firing rates in the $500 \mathrm{~ms}$ time 1015 window before animal's first lick. The decoding capability of each area was estimated by using 1016 varying numbers of randomly selected neurons that are recorded simultaneously in a session. As 1017 we included more neurons, sessions with insufficient number of neurons drop out in the decoding 1018 analysis. The classifier was trained on randomly selected $90 \%$ of the trials in each session and then 1019 tested on the remaining $10 \%$ of the trials. Only correct trials were used. The training/testing was 
1020 repeated 50 times for every given number of neurons and for all the sessions that may be included.

1021 The correct rates from the 50 repetitions were then averaged. When comparing performance in

1022 control and inactivation conditions (Fig. 4O-R), the classifiers were trained on correct control trials

1023 and tested on correct laser trials and held-out correct control trials.

1025 Imaging data analysis

1026 The raw images were first motion corrected with SIMA package (Kaifosh et al., 2014) (Release

1027 1.3.2) and verified manually. Regions of interest (ROIs) were selected automatically with 1028 constrained nonnegative matrix factorization (CNMF) (Pnevmatikakis et al., 2016). The CNMF

1029 algorithm infers the time-varying background and extracts smoothed $\Delta \mathrm{F} / \mathrm{F}$ signals, which were 1030 used for plotting only. For data analysis, we manually computed unfiltered $\Delta \mathrm{F} / \mathrm{F}$ traces as follows.

1031 We obtained the raw fluorescent trace of each ROI by applying the spatial component (ROI filter)

1032 on the image sequence. We then smooth the raw trace in each trial with a 1-s averaging window 1033 (boxcar) and take the minimal fluorescence value in the inter-trial interval as the baseline. The $1034 \Delta \mathrm{F} / \mathrm{F}$ signals were calculated by subtracting the baseline from the raw trace and dividing the 1035 difference by the baseline. We used the constrained deconvolution spike inference algorithm 1036 (FOOPSI) in the CNMF package to infer the spikes. The decay time constant was set at 0.7 second. 1037 The deconvolved activity was then smoothed using a gaussian filter over a five-element sliding 1038 window.

1040 We compared the means of the response to odors A and B during the sample and delay epochs 1041 using a Mann-Whitney $\mathrm{U}$ test. Each trial contributed a scalar value: the average $\Delta \mathrm{F} / \mathrm{F}$ signal from $1042 \mathrm{t}=0-2.5 \mathrm{~s}$ from onset of the sample odor. Neurons were classified as sample odor-selective if $P<$ 
10430.01 (two-tailed, not corrected for multiple comparisons). Test odor and choice selectivity were 1044 determined similarly using time bins of 2.5-4.0 s and 2.5-5.0 s from sample onset, respectively.

1045 Selectivity indices (SIs, Eq. 1) were computed from the same scalar values. The distributions of

1046 the sample odor SIs of ALM L2 neurons acquired with calcium imaging and those of ALM neurons

1047 sampled by electric recording were compared using a two-sample Kolmogorov-Smirnov test.

1049 The standardized odor and choice selectivity (Fig. 5A and S8D) were computed by dividing the

1050 absolute value of the difference between the mean $\Delta \mathrm{F} / \mathrm{F}$ responses to odor $\mathrm{A}$ and $\mathrm{B}$ (or match and 1051 non-match) by the combined standard deviation. The combined standard deviation is the square 1052 root of the sum of the variances for the $\Delta \mathrm{F} / \mathrm{F}$ responses to odor $\mathrm{A}$ and $\mathrm{B}$ (or match and non-match).

1054 To determine the duration of the calcium response, we first computed the mean and standard 1055 deviation of the baseline (the epoch before stimulus onset). Calcium transients were then identified 1056 as any response greater than 2 standard deviations away from the baseline and lasting for at least $1057100 \mathrm{~ms}$. The peak time is the time of the maximum calcium response. Only the calcium transients 1058 during the sample and delay epochs were considered in Fig. 5E. We used identical methods to 1059 determine the duration and peak time of the inferred spiking activity except that a minimal 1060 response duration was not required.

1062 To establish that a representation of sample odor in the population transient L2 neurons spans the 1063 entire sample plus delay, we measured the pairwise correlation in the peak times of the responses 1064 (Fig. S6A). Statistical significance of each r-value was established using Fisher z-transformed 1065 values and their s.e., without correction for multiple comparisons (gray shaded histograms, Fig. 
1066 S6B). We also estimated the distribution of correlation coefficients expected under the null 1067 hypothesis, using a shuffle control. The calculations are identical except each ordered pair from

1068 the two neurons comprises peak times from non-corresponding trials. The red dashed distribution

1069 (Fig. S6B) was estimated using 1000 iterations of this procedure.

1070

1071 For the decoding analysis (Fig. 6A-B), we trained a support vector machine (SVM) (Fan, Rong-

1072 En, 2008) using the calcium responses to classify sample odor identity (Fig. 6A) or

1073 match/nonmatch (Fig. 6B). The calcium responses were computed as the average $\Delta \mathrm{F} / \mathrm{F}$ in a sliding

1074 bin of $100 \mathrm{~ms}$ at $100 \mathrm{~ms}$ steps using 40 randomly selected neurons from each of the five cortical

1075 depths in ALM.

1076

1077 We characterized a trial-by-trial association between the Ca responses of L2 neurons and the

1078 likelihood that the mouse would make an error. The signals themselves are indirect measurements

1079 of neural activity and highly skewed. We therefore applied a variant of the choice probability

1080 measure based on ordinal statistics. We included 109 neurons that had statistically reliable

1081 preference for sample odor A or B using the integrated Ca signal over the sample and delay epochs.

1082 We included both correct and error trials in determining the sample odor selectivity, to avoid biases

1083 favoring neurons with larger responses in correct trials due to random fluctuation. For each neuron,

1084 the integrated signal was ranked and scored as a percentile ranking on $(0,1]$ using the trials in

1085 which the sample odor was the preferred odor to form the vector of trial-by-trial responses for each

1086 neuron, $\mathbf{r}_{n}^{\text {pref }}$, where the subscript identifies the neuron. The percentiles were assigned for each

1087 neuron independently (not the population). The percentiles from all the neurons were then pooled.

1088 We used the same procedure using the responses to each neuron's nonpreferred odor to form $\mathbf{r}_{n}^{\text {non }}$. 
1089 The heatmap in Fig. 6E was formed by parametrically varying the two criteria, $\kappa_{\text {pref }}$ and $\kappa_{\text {non }}$, to

1090 compute the proportion of errors when $\mathbf{r}^{\text {pref }}<\kappa_{\text {pref }}$ and $\mathbf{r}^{\text {non }}>\kappa_{\text {non }}$, using the combined data

1091 from all 109 neurons (i.e., concatenating across all $n$ ). To evaluated the null hypothesis that these

1092 responses have only a random association with the behavioral outcome, we conducted a simple

1093 logistic regression using the percentiles themselves:

$$
P_{\text {err }}=\left\{1+\exp \left(-\beta_{0}-\beta_{1} \mathbf{X}\right)\right\}^{-1}
$$

1095 Where $\mathrm{X}$ is the vector of the transformed percentiles:

$$
\mathbf{X}=\left(\begin{array}{c}
-\mathbf{r}^{\text {pref }}+1 \\
\mathbf{r}^{\text {non }}
\end{array}\right)
$$

1097 Note that the percentiles are simply reversed for $\mathbf{r}^{\text {pref }}$ so that the larger percentiles correspond to

1098 the weakest responses. We report the p-value associated with $\left\{H_{0}: \beta_{1}=0\right\}$.

1099

\section{Behavior/inhibition data analysis}

1101 Mouse performance (P) was reported as the fraction of correct responses in all trials. Animals may

1102 perform below chance (50\%) due to "no choice" trials (e.g., when they are challenged with novel

1103 C/D $\times$ C/D pairs; Fig. S1C). To assess the statistical reliability of photoinhibition on $\mathrm{P}$ we 1104 generated the distribution of $\log$ odds $(\mathcal{L})$ under the null hypothesis

$$
H_{0}: \mathcal{L} \equiv \log \frac{P_{\text {laser }}}{P_{\text {ctrl }}}=0
$$

1106 We randomly permuted the designations, correct/incorrect, among the laser and control trials 1107 within each trial type (AA/AB/BB/BA), repeating the process 10,000 times. From this distribution, 1108 we obtain the two-tailed probability of obtaining the observed log odds under $H_{0}$.

1110 To compare the effect of inactivation across tasks, we generated a distribution of $\mathcal{L}$ for each task 1111 by bootstrapping. The laser and control trials were re-sampled respectively with replacement 
1112 within each trial type (AA/AB/BB/BA). Repeating this process 10,000 times, we established a $t$ 1113 statistic from the means and variances of these distributions (degree of freedom based on the

1114 number of experimental trials). The reported significance reflects one-tailed comparisons.

1115

1116 We used the following logistic model to characterize an animal's bias. "No choice" trials in which

1117 animals did not respond were excluded in this analysis ( $<1 \%$ of trials).

$$
\begin{gathered}
P_{\text {left }}=\{1+\exp (-Q)\}^{-1} \\
Q=\beta_{0}+\beta_{1} S_{M}+\beta_{2} I_{\text {laser }}+\beta_{3} S_{M} I_{\text {laser }}
\end{gathered}
$$

1120 Where $P_{\text {left }}$ is the probably that the animal licks to the left port, $S_{M}$ is +1 or -1 if the trial is a match

1121 or non-match, respectively, and $I_{L}$ is 1 or 0 for laser on or off, respectively. The beta terms are

1122 fitted coefficients: $\beta_{0}$ quantifies the bias in favor of left on control trials in units of $\log$ odds; $\beta_{0}+$

$1123 \beta_{2}$ quantifies the bias in laser-on trials; $\beta_{1}$ quantifies how well the animal uses the match/non-

1124 match information to determine the direction of licking (i.e., sensitivity to condition) on the control

1125 trials; and $\beta_{1}+\beta_{3}$ quantifies the sensitivity in laser-on trials. In a well-trained animal, $\beta_{1}$ is always

1126 positive. $\beta_{2}$ is an estimate of the side bias induced by inactivation and $\beta_{3}$ is an estimate of the

1127 impairment on sensitivity by inactivation.

1129 When trials from all the animals are combined in this analysis, the bias coefficients could be 1130 underestimated $\left(\beta_{0}, \beta_{2}\right)$ because the bias for left or right is different across experiments. It is 1131 theoretically possible that underestimation of the laser induced bias, $\beta$, could lead to 1132 misattribution of this effect to a laser induced change in sensitivity, $\beta_{3}$. To address this, we first 1133 determined the bias of each animal in the laser condition by comparing the rate of correct match 1134 and non-match trials. Based on this bias, we designated left or right as the preferred lick port and 
match or non-match as the preferred trial for each animal. Then we combined the trials from all the animals to fit the following modified model:

$$
\begin{gathered}
P_{\text {pref }}=\{1+\exp (-Q)\}^{-1} \\
Q=\beta_{0}+\beta_{1} S_{\text {pref }}+\beta_{2} I_{\text {laser }}+\beta_{3} S_{\text {pref }} I_{\text {laser }}
\end{gathered}
$$

1139 Where $P_{\text {pref }}$ is the probability that the animal licks to the preferred port, and $S_{\text {pref }}$ is +1 or -1 if

1140 the trial is preferred or non-preferred, respectively. In this model, $\beta_{2}$ estimates the side bias induced

1141 by inactivation across all sessions. Importantly, if inactivation only biased the mouse to lick more

1142 to one port or the other - a different bias on each experiment - this procedure would fail to reject

1143 the null hypothesis, $\mathrm{H}_{0}: \beta_{3}=0$. We complemented this analysis using Monte Carlo methods to

1144 estimate the magnitude of impairment on the task that is not accounted for by a bias to the left or

1145 right lick port. We simulated datasets using the estimated $\beta$ coefficients and their standard error,

1146 while setting $\beta_{2}=0$. This recovers the proportion correct on the control trials and models the 1147 proportion correct on the inactivation trials, were no bias induced by the laser. In Fig. S4C, we 1148 show the distribution of impairments from 10,000 repetitions.

\section{Attractor network models}

1151 We constructed recurrent neural network models consisting of three areas (Fig. 7A). Each area $1152 a=1,2,3$ (representing Pir, intermediate areas, and ALM, respectively) contains $N=80$ units 1153 whose activities are represented by an $N$-dimensional vector $\mathbf{x}_{a}$ and follow the dynamics

$$
\tau \frac{d \mathbf{x}_{a}}{d t}=-\mathbf{x}_{a}+f\left(\mathbf{J}_{a} \mathbf{x}_{a}+\mathbf{J}_{a}^{F F} \mathbf{x}_{a-1}+\sigma \mathbf{\eta}_{a}(t)+\mathbf{b}_{\mathbf{a}}\right), a=1,2,3
$$

1155 The matrices $\mathbf{J}_{a}$ and $\mathbf{J}_{a}^{F F}$ represent recurrent input and feedforward input from the previous area, 1156 respectively. The vector $\mathbf{x}_{0}$ is two-dimensional, and its two elements are indicator variables (with 
1157 value 1 or 0$)$ representing the presence or absence of odors $\mathrm{A}$ and $\mathrm{B}$. The term $\sigma \boldsymbol{\eta}_{a}(t)$ represents

1158 independent white noise input with standard deviation $\sigma$. The vector $\mathbf{b}_{a}$ represents the bias inputs

1159 to each unit. The function $f$ is rectified-linear and the time constant $\tau$ (which represents combined

1160 membrane and synaptic time constants) equals $100 \mathrm{~ms}$.

1162 In each trial, the network receives sample and test odors (A or B) for $500 \mathrm{~ms}$ each, beginning at

1163 times $t=1$ and $3 \mathrm{~s}$. Each trial is drawn randomly from one of the four trial types. A readout of the

1164 network must classify the trial as match or non-match during the response period, from $t=3.5 \mathrm{~s}$

1165 to $t=4 \mathrm{~s}$. The readout is a softmax function of the ALM activity $\mathbf{x}_{3}$. At the beginning of each

1166 trial, the initial values of the $\mathbf{x}_{a}$ vectors are taken to be independent rectified Gaussian random

1167 variables with standard deviation 0.05 . Networks are simulated with a timestep of $20 \mathrm{~ms}$.

1169 The networks are trained through gradient descent with TensorFlow and the Adam optimizer

1170 (Agarwal et al., 2016; Kingma and $\mathrm{Ba}$, 2014) to minimize a loss $L=L_{\text {classifier }}+L_{\text {activity }}$

1171 determined by the classifier and an activity regularization term. Specifically, $L_{c l a s s i f i e r}$ in a given

1172 training epoch equals the summed cross-entropy loss between the classifier's output and the

1173 desired output (match or non-match) during the response period, averaged over a batch size of 50

1174 trials. The regularization term $L_{\text {activity }}=10^{-4} \cdot\left\langle\left\|\mathbf{r}_{a}\right\|^{2}\right\rangle$ is proportional to the $\ell_{2}$ norm of the

1175 activities averaged across units, time, areas, and batches. Every 50 epochs of training, the network

1176 is tested on 1000 trials to determine its classification accuracy, and training ceases when this

1177 accuracy exceeds $95 \%$. The noise $\sigma$ equals 0.05 during training and 0.1 during this testing phase.

1178 The learning rate of the optimizer decreases logarithmically from $10^{-3}$ to $10^{-4}$ over 1000 epochs.

1179 Networks which do not reach the 95\% criterion accuracy after these 1000 epochs are discarded. 
1181 At the beginning of training, all recurrent and feedforward weights $\mathbf{J}_{a}, \mathbf{J}_{a}^{F F}$ are initialized as

1182 independent random Gaussian variables with standard deviation $1 / \sqrt{N}$ (except for $\mathbf{J}_{0}^{F F}$, which has

1183 standard devation $1 / \sqrt{2}$ because of the dimension of $\mathbf{x}_{0}$ ). Half of the feedforward weights are then

1184 randomly set equal to zero, representing sparser connections across areas versus within areas. The

1185 softmax classifier weights are initialized with standard deviation $2 / \sqrt{N}$, and the biases $\mathbf{b}_{a}$ are

1186 initialized to zero. We assume that only $\mathbf{J}_{a}$ and $\mathbf{b}_{a}$ are learned. All other variables are fixed during 1187 training.

To generate the performance curves in Fig. 7B, 60 trained networks were tested with $\sigma=0.2$ under four conditions. In the control condition, the dynamics were identical to those described above. In the other conditions, which mimic ALM inactivation during different periods of the task,

1192 an inhibitory input was applied to ALM units during the sample and early delay periods (the $1 \mathrm{~s}$ 1193 following sample onset), the late delay period (the final $1 \mathrm{~s}$ of the delay period), or the entire 1194 sample and delay periods. The inhibitory input was equivalent to reducing the biases of all ALM 1195 units $\mathbf{b}_{3}$ by 5 . To generate the curve for networks without ALM persistence, modifications of $\mathbf{J}_{a}$ were restricted to only $\mathbf{J}_{1}$ and $\mathbf{J}_{2}$ while $\mathbf{J}_{3}$ was held fixed, so that the training algorithm could not

1197 learn to implement attractor dynamics in $\operatorname{ALM}(a=3)$. Conversely, for networks with only ALM 1198 persistence, training was restricted to only $\mathbf{J}_{3}$, while $\mathbf{J}_{1}$ and $\mathbf{J}_{2}$ were held fixed. 
1204 Devin, M., Ghemawat, S., et al. (2016). TensorFlow: Large-Scale Machine Learning on

1205 Heterogeneous Distributed Systems. ArXiv 1603.04467, [cs].

1206 Fan, Rong-En, et al (2008). LIBLINEAR: A library for large linear classification. J. Mach.

1207 Learn. Res. 9, 1871-1874.

1208 Guo, Z., Li, N., Huber, D., Ophir, E., Gutnisky, D., Ting, J., Feng, G., and Svoboda, K. (2014a).

1209 Flow of cortical activity underlying a tactile decision in mice. Neuron 81, 179-194.

1210 Guo, Z. V, Hires, S.A., Li, N., O’Connor, D.H., Komiyama, T., Ophir, E., Huber, D., Bonardi, 1211 C., Morandell, K., Gutnisky, D., et al. (2014b). Procedures for behavioral experiments in head-

1212 fixed mice. PLoS One 9, e88678.

1213 Kaifosh, P., Zaremba, J.D., Danielson, N.B., and Losonczy, A. (2014). SIMA: Python software 1214 for analysis of dynamic fluorescence imaging data. Front. Neuroinform. 8, 80.

1215 Kingma, D.P., and Ba, J.L. (2014). Adam: A Method for Stochastic Optimization. ArXiv 1216 1412.6980, [cs].

1217 Pachitariu, M., Steinmetz, N.A., Kadir, S.N., Carandini, M., and Harris, K.D. (2016). Fast and 1218 accurate spike sorting of high-channel count probes with KiloSort. NIPS Proc. 4448-4456.

1219 Pnevmatikakis, E.A., Soudry, D., Gao, Y., Machado, T.A., Merel, J., Pfau, D., Reardon, T., Mu, 1220 Y., Lacefield, C., Yang, W., et al. (2016). Simultaneous Denoising, Deconvolution, and 1221 Demixing of Calcium Imaging Data. Neuron 89, 299.

1222 Root, C.M., Denny, C.A., Hen, R., and Axel, R. (2014). The participation of cortical amygdala in 
1223 innate, odour-driven behaviour. Nature 515, 269-273.

1224 Rossant, C., Kadir, S.N., Goodman, D.F.M., Schulman, J., Hunter, M.L.D., Saleem, A.B.,

1225 Grosmark, A., Belluscio, M., Denfield, G.H., Ecker, A.S., et al. (2016). Spike sorting for large,

1226 dense electrode arrays. Nat. Neurosci. 19, 634-641. 